\title{
Overlay Spectrum Sharing using Improper Gaussian Signaling
}

\author{
Osama Amin, Senior Member, IEEE, Walid Abediseid, Member, IEEE, and Mohamed-Slim Alouini, Fellow, IEEE
}

\begin{abstract}
Improper Gaussian signaling (IGS) scheme has been recently shown to provide performance improvements in interference limited networks as opposed to the conventional proper Gaussian signaling (PGS) scheme. In this paper, we implement the IGS scheme in overlay cognitive radio system, where the secondary transmitter broadcasts a mixture of two different signals. The first signal is selected from the PGS scheme to match the primary message transmission. On the other hand, the second signal is chosen to be from the IGS scheme in order to reduce the interference effect on the primary receiver. We then optimally design the overlay cognitive radio to maximize the secondary link achievable rate while satisfying the primary network quality of service requirements. In particular, we consider full and partial channel knowledge scenarios and derive the feasibility conditions of operating the overlay cognitive radio systems. Moreover, we derive the superiority conditions of the IGS schemes over the PGS schemes supported with closed form expressions for the corresponding power distribution and the circularity coefficient and parameters. Simulation results are provided to support our theoretical derivations.
\end{abstract}

Index Terms-Spectrum sharing, overlay cognitive radio, improper Gaussian signaling, interference channel, power allocation, circularity coefficient, pseudo variance.

\section{INTRODUCTION}

The widespread growth in wireless technologies encourages investors and developers to offer new wireless services, applications, and equipment. Such a tremendous increase of wireless traffic with ubiquitous connectivity demand increases the bandwidth requirement exponentially. However, the current fixed policies of spectrum allocation lead to inefficient use of the spectrum and prevent accommodating new users. Motivated by this spectrum scarcity problem, different communication regulators, such as the Federal Communications Commission (FCC) and Office of Communication (Ofcom), persuade deploying new technologies to enable efficient spectrum usage by dynamic spectrum access. The main idea is to create unlicensed (secondary) networks that can coexist with licensed (primary) networks in order to enable accepting additional users without disrupting the licensed network operation [1].

Cognitive radio is the term used to capture dynamic spectrum access techniques, which are known as underlay, overlay and interweave [1]. The underlay cognitive radio imposes limitations on the unlicensed users power so that its power received at the licensed receiver does not affect the licensed user target quality-of-service (QoS). The overlay cognitive radio uses part of its resources to assist the licensed network in delivering its messages, while the other part of the resources is used to

The authors are with Computer, Electrical, and Mathematical Sciences and Engineering (CEMSE) Divison, King Abdullah University of Science and Technology (KAUST), Thuwal, Makkah Province, Saudi Arabia. Email: \{osama.amin, walid.abediseid, slim.alouini\}@kaust.edu.sa. deliver its messages without affecting the primary link QoS. In this technique, the interference at the secondary network can be canceled using the shared primary network message and the availability of channel state information (CSI). Last but not least, the interweave cognitive radio operates by scanning the licensed spectrum and searching for the unused spectrum holes in order to transmit its message through it as long as it is available [1], [2]. Therefore, different from interweave spectrum sharing techniques, which does not interfere with the primary network, both underlay and overlay cognitive radio can apply interference on the primary network. Therefore, interference management becomes a key enabler for realizing dense networks that uses the same spectrum.

One way to improve the overall performance of the interference limited networks is to design the statistical characteristics of the signals. Improper Gaussian signaling (IGS) scheme has been shown to improve the achievable rate of interference limited networks compared with its counterpart proper Gaussian signaling (PGS) scheme. In contrast to the PGS scheme which assumes independent real and imaginary signal components with equal power, the IGS scheme relaxes these assumptions introducing a general Gaussian signaling scheme [3]-[5]. PGS has been proven to be the optimal signaling scheme for pointto-point channels with additive white Gaussian noise (AWGN), while the optimal performance does not hold for interference limited networks. On the other hand, IGS scheme achieves superior rate improvements for systems not supported with interference cancellation techniques and deal with interference as noise. IGS scheme is employed in different interference scenarios such as two user single input single output interference channel [6], $k$-user multiple input single output interference channel [7], full duplex relaying [8], alternate relaying [9] and underlay cognitive radio [10]-[16].

IGS scheme has proved its superiority in enhancing the underlay cognitive radio performance through reducing the interference effect on the primary user [10]-[16]. In underlay cognitive radio system, there is no access on designing the licensed network signals or systems. Furthermore, a common and reasonable assumption of the primary network is the operation as if there no other user shares the spectrum. Thus, the primary network is assumed to employ PGS scheme since it accesses its dedicated licensed channels [10]-[16]. On the other hand, the secondary network uses IGS scheme to reduce its interference on the primary network. The results in [10][16] demonstrated benefits for adopting IGS as long as the PT link is not fully loaded especially when the secondary network exists near the receivers of the primary network. The merits can be reaped using global knowledge of instantaneous CSI [10], [11], [14] or even using average CSI [12], [13], [15], [16]. 
Moreover, IGS grants performance improvement for secondary networks when they share the spectrum with half-duplex transmissions [10], [12] or full-duplex transmissions [14][16]. As for overlay cognitive radio, to the best of authors' knowledge, IGS scheme has not yet been implemented.

Overlay cognitive radio is different from the underlay cognitive radio in offering a coordination between the secondary network and the primary network to deliver the latter data. Throughout the paper, we follow a similar coordination model in [2], [17], where the secondary network delivers parts of the primary message to the intended primary user while transmitting its own message simultaneously in the same band. Thus, the secondary network broadcasts a superposition code of two different messages and design its system parameters to meet the required QoS at the primary network side. Achieving benefits from the asymmetric statistical signal structure in IGS can not be known especially that PGS scheme is shown to achieve the optimal performance of other systems that use superposition code, such as partial decode and forward full duplex relaying systems [18].

In this paper, we study the impact of employing the IGS scheme on the achievable rate of the overlay cognitive radio system. Being the first study of adopting IGS scheme in overlay spectrum sharing, we consider a simplified system model with a single user in each network in order to explore possible benefits before delving into multiple users and/or large sale networks ${ }^{1}$. The secondary network broadcasts a mixture of two messages, the first is of PGS type for the primary user and the second is of IGS type for the secondary user. The primary message is chosen to be PGS in order to match the signal of the primary network, and to avoid any decoding burden at its receiver side. On the other hand, the second message is chosen to be of IGS type to reduce the interference effect on the primary network receiver. The contribution of this paper is summarized as follows:

- Adopt IGS in overlay cognitive radio system to maximize the secondary network achievable rate while satisfying a minimum QoS of the primary network. With global knowledge of CSI at the secondary network side, the QoS is measured by a minimum rate requirement. On the other hand, with partial CSI knowledge, the QoS is measured by a maximum outage probability for a target rate.

- For both cases, i.e., global and partial CSI knowledge of CSI at the secondary network side, we prove the following:

- Feasibility conditions of overlay cognitive radio systems operation.

- Necessary and sufficient conditions needed for IGS based systems to provide improvement over PGS based systems.

\footnotetext{
${ }^{1}$ It is worth to mention that the proposed approach can be directly implemented in the following systems: 1) multiple primary transmitters that use orthogonal time/frequency slots for communication and share the spectrum with different secondary users. 2) multiple primary transmitters that suffers from inter-cell interference and share the spectrum with different secondary users, where the interference sources are assumed be fixed as an additive noise term similar to [13] and [14].
}

- Optimal signal design for both PGS and IGS based schemes to maximize the achievable rate of the secondary network while satisfying the primary network QoS.

The rest of the paper is organized as follows. In Section II, we describe the overlay cognitive radio system model. Section III studies the design of signal characteristics for overlay cognitive radio assuming full CSI knowledge at the secondary. Then, we extend the design problem assuming partial CSI at the secondary network side in Section IV. Then, we validate the performance improvement through simulation examples in Section V. Finally, we conclude the paper in Section VI.

Notations: |.| denotes the absolute value operator, $\mathbb{E}[$. denotes the statistical expectation operator and $\bar{x}$ is the mean value of $\mathrm{x}$, i.e., $\bar{x}=\mathbb{E}[x]$.

\section{SySTEM DESCRIPTION}

\section{A. Preliminaries}

The PGS scheme can be fully characterized by the variance assuming it is zero mean signal. On the other hand, this characterization is not sufficient for the IGS scheme. Therefore, we introduce in the following discussion some definitions to differentiate between these two signaling schemes. To this end, we consider a zero mean scalar random variable $x$ whose conventional variance is defined as $\sigma_{x}^{2}=\mathbb{E}\left[|x|^{2}\right]$, and its pseudo-variance is defined as $\tilde{\sigma}_{x}^{2}=\mathbb{E}\left[x^{2}\right][3]$.

Definition 1: [3], [4] A complex random variable is called proper if its pseudo-variance is equal to zero, otherwise it is called improper.

Definition 2: [10] The impropriety degree of $x$ is measured by the circularity coefficient that is defined as $\mathcal{C}_{x}=\left|\tilde{\sigma}_{x}^{2}\right| / \sigma_{x}^{2}$, where $0 \leq \mathcal{C}_{x} \leq 1, \mathcal{C}_{x}=0$ denotes proper signal and $\mathcal{C}_{x}=1$ denotes maximally improper signal.

The IGS scheme adds a new dimension that does not exist in the PGS scheme giving an additional degree of freedom in the system design, which allows improving system performance or keep it the same in the worst case.

\section{B. Overlay Cognitive Radio System}

Consider the wireless communication setup depicted in Fig. 1, which consists of a primary licensed network and a secondary unlicensed network. The licensed network consists of a base station, which is the primary transmitter (PT) and a single user that acts as a primary receiver (PR). The secondary network consists of a secondary transmitter (ST) represented in the access point in Fig. 1 and a single user acting as the secondary receiver (SR). The ST is assumed to be connected to the PT via a robust backhaul communication link such as optical fibers in order to have access to the PT messages and support its transmission when needed ${ }^{2}$. Therefore, the ST can

\footnotetext{
${ }^{2}$ If the backhaul link is not available, wireless cooperative techniques can be used to share the primary message and other related information, thus the primary network performance will be affected by the quality of the wireless link. In case of the unavailability of the primary message and/or the CSI, orthogonal spectrum sharing using cooperative techniques become an alternative solution as in [19], otherwise, the primary link can impose interference on the secondary link that need to be mitigated.
} 
coordinate with the PT and operate as a distributed antenna node for the primary network to extend the primary network coverage and improve its performance. In the same time, the ST can virtually create a secondary network that does not affect the primary network QoS. Furthermore, the ST can cancel the interference from the PT at the SR side based on its knowledge of the primary message and the CSI using dirty paper coding technique ${ }^{3}$ [21]. Similar scenario occurs in systems that coordinate multi-point transmissions and use the backhaul links to share their data and CSI to deliver the required data while canceling the inter-cell interference [22].

In the overlay cognitive radio model, the ST allocates $\alpha p_{\mathrm{s}}$ of its total power $p_{\mathrm{s}}$, where $0 \leq \alpha \leq 1$, to send a part of the PT message to the PR which receives another part of the message through the primary link. The remaining ST power, i.e., $(1-\alpha) p_{\mathrm{s}}$, is used to transmit the secondary message. The value of $\alpha$ is computed to improve the secondary network QoS while satisfying the minimum QoS of the primary network. Since the primary network is accessing its licensed band, we assume that it is not aware of the existence of the secondary network. As such, the primary network considers the PGS scheme as it is the optimal scheme in its point-to-point AWGN channel. On the other hand, since the secondary network causes interference at the PR, the transmitted secondary message $x_{\mathrm{s}}$ is chosen from the IGS scheme. Adopting IGS scheme increases the design degrees of freedom and allows to reduce the interference impact on the primary network.

The received signal at the PR, $y_{\mathrm{p}}$, is expressed as

$$
y_{\mathrm{p}}=\sqrt{p_{\mathrm{p}}} h_{\mathrm{p}} x_{\mathrm{p}, 1}+\sqrt{\alpha p_{\mathrm{s}}} g_{\mathrm{s}} x_{\mathrm{p}, 2}+\sqrt{(1-\alpha) p_{\mathrm{s}}} g_{\mathrm{s}} x_{\mathrm{s}}+n_{\mathrm{p}},
$$

where $x_{\mathrm{p}, 1}$ denotes the transmitted primary message part from the PT with power $p_{\mathrm{p}}, x_{\mathrm{p}, 2}$ denotes a different transmitted primary message part from the ST with power $\alpha p_{\mathrm{s}}$ that is independent of $x_{\mathrm{p}, 1}, h_{\mathrm{p}}$ and $g_{\mathrm{s}}$ are the fading channel coefficients of the primary link and the cross link from the ST to the PR, respectively, and $n_{\mathrm{p}}$ is the AWGN at the PR end with zero mean and variance $\sigma_{n}^{2}$.

The received signal at the $\mathrm{SR}, y_{\mathrm{s}}$, is written as

$$
y_{\mathrm{s}}=\sqrt{(1-\alpha) p_{\mathrm{s}}} h_{\mathrm{s}} x_{\mathrm{s}}+\sqrt{p_{\mathrm{p}}} g_{\mathrm{p}} x_{\mathrm{p}, 1}+\sqrt{\alpha p_{\mathrm{s}}} h_{\mathrm{s}} x_{\mathrm{p}, 2}+n_{\mathrm{s}}
$$

where $h_{\mathrm{s}}$ and $g_{\mathrm{p}}$ are the fading channel coefficients of the secondary direct link and the interference link from PT to the $\mathrm{SR}$, respectively, and $n_{\mathrm{S}}$ is the AWGN at the SR end with zero mean and variance $\sigma_{n}^{2}$.

\section{Achievable Rates}

In this section, we express the achievable rate for overlay cognitive radio system with PGS and IGS schemes. As pointed in (1), the primary transmitter sends $x_{\mathrm{p}, 1}$ that is independent of the secondary transmitter message $x_{\mathrm{p}, 2}$. Since both signals are independent, then the overall transmitted signaling scheme can be seen as a two-level superposition coding scheme, in

\footnotetext{
${ }^{3}$ As for the DPC deployment, the main challenges are sharing the primary message that can be done using the backhaul and estimating the CSI, which can be done at the SR end with the knowledge of primary data and pilot structure. As for the deployment, different practical hardware realization can be implemented such as FPGA [20].
}

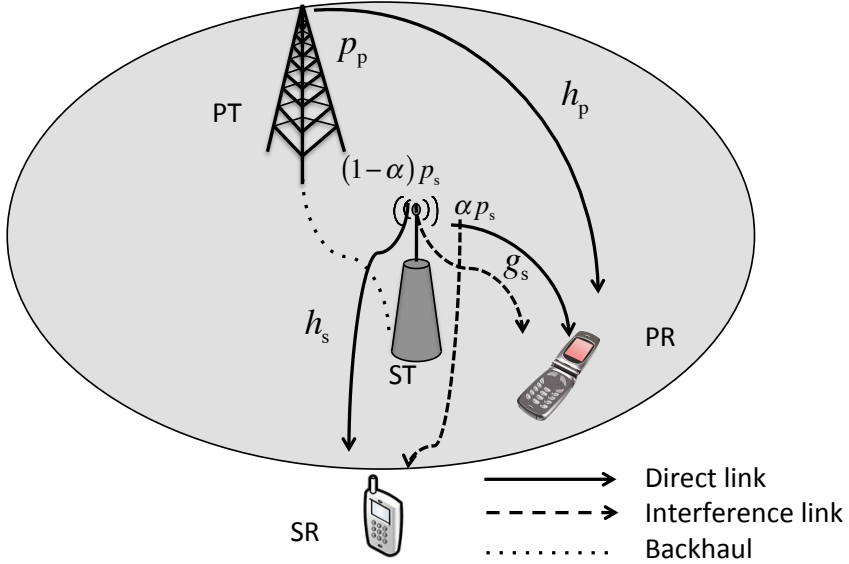

Fig. 1. Overlay cognitive radio system model.

which two independent messages are encoded at different rates and successively decoded at the receiver. Since the primary receiver must decode both $x_{\mathrm{p}, 1}$ and $x_{\mathrm{p}, 1}$, then its achievable rate, denoted by $R_{\mathrm{p}}$ would now be the sum of the rates provided by $x_{\mathrm{p}, 1}$ and $x_{\mathrm{p}, 1}$. Based on the achievable rate formula presented in [6, eq. (30)], the expression of the achievable rate of the primary network when the ST uses IGS scheme can be shown to be given by:

$$
\begin{aligned}
& R_{\mathrm{p}}\left(\alpha, \mathcal{C}_{x}\right)=\log _{2}\left(1+\frac{p_{\mathrm{p}} \mathcal{H}_{\mathrm{p}}+\alpha p_{\mathrm{s}} \mathcal{G}_{\mathrm{s}}}{(1-\alpha) p_{\mathrm{s}} \mathcal{G}_{\mathrm{s}}+1}\right) \\
&+\frac{1}{2} \log _{2}\left(\frac{1-\mathcal{C}_{y_{\mathrm{p}}}^{2}}{1-\mathcal{C}_{I_{\mathrm{p}}}^{2}}\right)
\end{aligned}
$$

where $\mathcal{H}_{\mathrm{p}}=\left|h_{\mathrm{p}}\right|^{2} / \sigma_{n}^{2}$ and $\mathcal{G}_{\mathrm{s}}=\left|g_{\mathrm{s}}\right|^{2} / \sigma_{n}^{2}$, and $\mathcal{C}_{y_{\mathrm{p}}}$ and $\mathcal{C}_{I_{\mathrm{p}}}$ are the circularity coefficients of the received and interferenceplus-noise signals at the PU, respectively. After few manipulation, $R_{\mathrm{p}}\left(\alpha, \mathcal{C}_{x}\right)$, can be rewritten as

$$
\begin{aligned}
R_{\mathrm{p}}\left(\alpha, \mathcal{C}_{x}\right)= & \frac{1}{2} \log _{2}(1+ \\
& \left.\frac{\left(2+(2-\alpha) p_{\mathrm{s}} \mathcal{G}_{\mathrm{s}}+p_{\mathrm{p}} \mathcal{H}_{\mathrm{p}}\right)\left(\alpha p_{\mathrm{s}} \mathcal{G}_{\mathrm{s}}+p_{\mathrm{p}} \mathcal{H}_{\mathrm{p}}\right)}{\left((1-\alpha) p_{\mathrm{s}} \mathcal{G}_{\mathrm{s}}+1\right)^{2}-\left((1-\alpha) p_{\mathrm{s}} \mathcal{G}_{\mathrm{s}} \mathcal{C}_{x}\right)^{2}}\right) .
\end{aligned}
$$

One can note from (4) that $R_{\mathrm{p}}\left(\alpha, \mathcal{C}_{x}\right)$ is a monotonically increasing function in $\mathcal{C}_{x}$. Therefore, using IGS scheme improves the achievable rate in general. Substituting $\mathcal{C}_{x}=0$ in (4), the achievable rate of the primary network when the ST adopts only the PGS scheme can be expressed as

$$
R_{\mathrm{p}}(\alpha, 0)=\log _{2}\left(1+\frac{\alpha p_{\mathrm{s}} \mathcal{G}_{\mathrm{s}}+p_{\mathrm{p}} \mathcal{H}_{\mathrm{p}}}{(1-\alpha) p_{\mathrm{s}} \mathcal{G}_{\mathrm{s}}+1}\right) .
$$

As for the secondary link, we assume the ST has access to the primary messages $\left(x_{\mathrm{p}, 1}\right.$ and $\left.x_{\mathrm{p}, 2}\right)$ in addition to the CSI, the interference due to the primary message can be canceled using dirty paper coding [21]. Therefore, following a similar approach as in the primary network case, the achievable rate 
of the secondary link, denoted by $R_{\mathrm{s}}$, based on IGS scheme can be expressed as

$$
\begin{aligned}
R_{\mathrm{S}}\left(\alpha, \mathcal{C}_{x}\right)=\frac{1}{2} \log _{2}(1 & +2(1-\alpha) p_{\mathrm{s}} \mathcal{H}_{\mathrm{s}} \\
& \left.+\left(1-\mathcal{C}_{x}^{2}\right)\left((1-\alpha) p_{\mathrm{s}} \mathcal{H}_{\mathrm{s}}\right)^{2}\right),
\end{aligned}
$$

where $\mathcal{H}_{\mathrm{s}}=\left|h_{\mathrm{s}}\right|^{2} / \sigma_{n}^{2}$. Although the use of IGS scheme to transmit the secondary message improves the achievable rate of the primary link, it reduces the rate of the secondary link. When $\mathcal{C}_{x}=0 R_{\mathrm{s}}$, reduces simply to the well known achievable rate expression

$$
R_{\mathrm{s}}(\alpha, 0)=\log _{2}\left(1+(1-\alpha) p_{\mathrm{s}} \mathcal{H}_{\mathrm{s}}\right) .
$$

\section{Overlay Cognitive Radio System Design With FULL-CSI}

In this section, we optimize the overlay cognitive radio statistical signal parameters in order to improve the QoS of the secondary network while maintaining a target QoS for the primary network. As a metric to measure the QoS, we use a minimum rate requirement for the primary network, denoted by $R_{\min }$ and the achievable rate for the secondary transmission denoted by $R_{\mathrm{S}}\left(\alpha, \mathcal{C}_{x}\right)$. First, we optimize $\alpha$ at the ST when the corresponding two messages transmitted are of the PGS scheme. Then, both values of $\alpha$ and $\mathcal{C}_{x}$ are jointly optimized at ST when the second message of the ST-SR link is of IGS scheme.

\section{A. Proper Gaussian Signaling based Design}

The achievable rate of the secondary link based on the PGS scheme can be maximized by solving the following optimization problem

$$
\begin{array}{cl}
\max & R_{\mathrm{s}}(\alpha, 0) \\
\text { subject to } & R_{\mathrm{p}}(\alpha, 0) \geq R_{\min } \\
& 0 \leq \alpha \leq 1 .
\end{array}
$$

To obtain the solution of (8), we first study the feasibility condition of overlay spectrum sharing using the following theorem.

Theorem 1. In an overlay cognitive radio system with a minimum rate requirement $R_{\min }$ for the primary network, the secondary network can transmit information to the $S R$ if and only if $R_{\min }<R_{0}$, where $R_{0}$ is given by

$$
R_{0}=\log _{2}\left(1+p_{\mathrm{s}} \mathcal{G}_{\mathrm{s}}+p_{\mathrm{p}} \mathcal{H}_{\mathrm{p}}\right)
$$

Proof. The maximum achievable rate of the primary network is achieved when the ST is fully dedicated to the primary network and is computed from (5) at $\alpha=1$ that reduces to (9). Therefore, if $R_{\min }<R_{0}$, the $\mathrm{ST}$ uses a portion of its power budget to send the primary message and the rest to send the secondary message while satisfying the primary network QoS. It is worthy to emphasize that when $\alpha=1$, PGS become the optimal signaling scheme since it is a point-to-point AWGN channel.

It is worth to emphasize that Theorem 1 represents the feasibility condition of overlay cognitive radio system operation regardless the type of secondary message signaling scheme whether it is PGS or IGS. When the feasibility condition is not valid, the primary link is not reliable and fails to achieve its required minimum rate even if the ST is assisting the primary network with its full power budget. In this scenario, we assume that the ST assigns its total power to improve the primary network QoS, although it is considered in outage case. In the next theorem, we consider a feasible overlay cognitive radio, then optimally design $\alpha$ to maximize $R_{\mathrm{s}}(\alpha, 0)$ while satisfying a minimum rate requirement $R_{\min }$ for the primary network.

Theorem 2. In a feasible overlay cognitive radio system with PGS scheme adopted at both the primary and secondary networks, the ST power $p_{\mathrm{s}}$ is optimally divided between the primary message and the secondary message as $\alpha_{\mathrm{PGS}} p_{\mathrm{S}}$ and $\left(1-\alpha_{\mathrm{PGS}}\right) p_{\mathrm{s}}$, respectively, with $\alpha_{\mathrm{PGS}}$ is expressed as

$$
\alpha_{\mathrm{PGS}}=\left\{\begin{array}{cc}
\rho & R_{\mathrm{p}, 1} \leq R_{\min } \leq R_{0}, \\
0 & R_{\min }<R_{\mathrm{p}, 1},
\end{array}\right.
$$

where $\rho$ is defined as

$$
\rho=1-\frac{1}{p_{\mathrm{s}} \mathcal{G}_{\mathrm{s}}}\left(\frac{p_{\mathrm{s}} \mathcal{G}_{\mathrm{s}}+p_{\mathrm{p}} \mathcal{H}_{\mathrm{p}}+1}{2^{R_{\min }}}-1\right),
$$

and $R_{\mathrm{p}, 1}$ is expressed as

$$
R_{\mathrm{p}, 1}=\log _{2}\left(1+\frac{p_{\mathrm{p}} \mathcal{H}_{\mathrm{p}}}{p_{\mathrm{s}} \mathcal{G}_{\mathrm{s}}+1}\right) .
$$

Proof. Consider the optimization problem in (8) and simplify it by rewriting the first constraint in terms of $\alpha$ using the the monotonic increasing characteristic of the logarithmic function in the objective function obtaining equivalent optimization problem

$$
\begin{array}{cl}
\min & \alpha \\
\text { subject to } & \alpha \geq \rho \\
& 0 \leq \alpha \leq 1 .
\end{array}
$$

Under the assumption of feasible overlay cognitive radio system reported in Theorem 1, the solution of (13) reduces to $\rho$ as long as $\rho>0$ or equivalently $R_{\min }>R_{\mathrm{p}, 1}$, which can be proven after simple manipulation. On the other hand, if $\rho<0$ (or equivalently $R_{\min }<R_{\mathrm{p}, 1}$ ), the solution reduces to $\alpha=0$. The latter case indicates that the primary link from PT to the PR is very strong which allows achieving the minimum rate requirement not only without the help of ST, but also under a full interference load from the ST link.

According to the Theorems 1 and 2, we have two cases where the IGS can not provide any benefit. First, for $R_{\min }>$ $R_{0}$, where introducing interference to the primary transmission is not permitted since the primary transmission link suffers from unacceptable outage performance. Second, for $R_{\min } \leq$ $R_{\mathrm{p}, 1}$, where the full insertion of the secondary network load does not affect the primary network QoS. Therefore, the optimal signaling scheme for the ST is PGS scheme since the secondary network does not suffer from any interference thanks to dirty paper coding and become equivalent to pointto-point AWGN communication channel. Therefore, IGS can introduce benefit in the design problem for $R_{\mathrm{p}, 1}<R_{\min }<R_{0}$ as will be discussed in the following subsection. 


\section{B. Improper Gaussian Signaling based Design}

For the IGS based scheme, we optimize $\alpha$ and $\mathcal{C}_{x}$ based on the following optimization problem

$$
\begin{array}{cl}
\max & R_{\mathrm{s}}\left(\alpha, \mathcal{C}_{x}\right) \\
\text { subject to } & R_{\mathrm{p}}\left(\alpha, \mathcal{C}_{x}\right) \geq R_{\min } \\
& 0 \leq \alpha \leq 1 \\
& 0 \leq \mathcal{C}_{x} \leq 1
\end{array}
$$

By solving the IGS optimization design problem (14), we obtain the necessary and sufficient conditions to use IGS for the secondary message signal based on the following theorem.

Theorem 3. In a feasible overlay cognitive radio system with $P G S$ scheme adopted at the primary network, adopting IGS scheme at the secondary network improves its achievable rate performance compared with PGS based scheme if and only if:

1) $\mathcal{G}_{\mathrm{s}}>\mathcal{H}_{\mathrm{s}}$

2) $R_{\mathrm{I}, 1}<R_{\min }<R_{0}$, where

$$
\begin{array}{r}
R_{\mathrm{I}, 1}=\log _{2}\left(\frac{(2 / 3)^{1 / 3}}{\left(9 \beta+\sqrt{3} \sqrt{-4+27 \beta^{2}}\right)^{1 / 3}}\right. \\
\left.+\frac{\left(9 \beta+\sqrt{3} \sqrt{-4+27 \beta^{2}}\right)^{1 / 3}}{2^{1 / 3} 3^{2 / 3}}\right),
\end{array}
$$

with $\beta=p_{\mathrm{s}} \mathcal{H}_{\mathrm{s}}\left(p_{\mathrm{s}} \mathcal{G}_{\mathrm{s}}+p_{\mathrm{p}} \mathcal{H}_{\mathrm{p}}+1\right) /\left(p_{\mathrm{s}} \mathcal{G}_{\mathrm{s}}-p_{\mathrm{s}} \mathcal{H}_{\mathrm{s}}\right)$.

Proof. Consider the optimization problem (14) and simplify the objective function by using the logarithmic function characteristic and express it in terms of its argument. Then, we simplify the constraint obtaining the following equivalent optimization problem

$$
\begin{array}{cl}
\max & f\left(\alpha, \mathcal{C}_{x}\right) \\
\text { subject to } & f\left(\alpha, \mathcal{C}_{x}\right) \leq f_{0}(\alpha) \\
& 0 \leq \alpha \leq 1 \\
& 0 \leq \mathcal{C}_{x} \leq 1,
\end{array}
$$

where $f\left(\alpha, \mathcal{C}_{x}\right)$ is expressed as

$$
f\left(\alpha, \mathcal{C}_{x}\right)=2(1-\alpha)+\left(1-\mathcal{C}_{x}^{2}\right)(1-\alpha)^{2} p_{\mathrm{s}} \mathcal{H}_{\mathrm{s}}
$$

and $f_{0}(\alpha)$ is defined as

$$
\begin{aligned}
f_{0}(\alpha)= & \frac{p_{\mathrm{s}} \mathcal{H}_{\mathrm{s}}\left(\alpha p_{\mathrm{s}} \mathcal{G}_{\mathrm{s}}+p_{\mathrm{p}} \mathcal{H}_{\mathrm{p}}\right)\left((2-\alpha) p_{\mathrm{s}} \mathcal{G}_{\mathrm{s}}+2+p_{\mathrm{p}} \mathcal{H}_{\mathrm{p}}\right)}{\left(2^{2 R_{\min }}-1\right)\left(p_{\mathrm{s}} \mathcal{G}_{\mathrm{s}}\right)^{2}} \\
& +2(1-\alpha)-\frac{p_{\mathrm{s}} \mathcal{H}_{\mathrm{s}}\left(2(1-\alpha) p_{\mathrm{s}} \mathcal{G}_{\mathrm{s}}+1\right)}{\left(p_{\mathrm{s}} \mathcal{G}_{\mathrm{s}}\right)^{2}}
\end{aligned}
$$

One can prove that the objective function in (16) is monotonically decreasing in $\alpha$ and $\mathcal{C}_{x}$ [23]. Furthermore, one can show that the first constraint in (16) can be equivalently expressed as $\alpha \geq a\left(\mathcal{C}_{x}\right)$, where $a\left(\mathcal{C}_{x}\right)$ is defined as

$$
\begin{aligned}
& a\left(\mathcal{C}_{x}\right)=1-\frac{1}{\left(1-\mathcal{C}_{x}^{2}\left(1-\frac{1}{2^{2 R_{\min }}}\right)\right) p_{\mathrm{s}} \mathcal{G}_{\mathrm{s}}}[-1+ \\
& \left.\sqrt{1+\left(1-\mathcal{C}_{x}^{2}\left(1-\frac{1}{2^{2 R_{\min }}}\right)\right)\left(\left(\frac{p_{\mathrm{s}} \mathcal{G}_{\mathrm{s}}+p_{\mathrm{p}} \mathcal{H}_{\mathrm{p}}+1}{2^{R_{\min }}}\right)^{2}-1\right)}\right] .
\end{aligned}
$$

Therefore, the optimization problem solution exists at $\alpha=$ $a\left(\mathcal{C}_{x}\right)$ or equivalently at $f\left(\alpha, \mathcal{C}_{x}\right)=f_{0}(\alpha)$. According to this fact, we simplify the optimization problem (16) by changing the objective function to be $f_{0}(\alpha)$ obtaining the following equivalent optimization problem instead

$$
\begin{array}{cl}
\max & f_{0}(\alpha) \\
\text { subject to } & f\left(\alpha, \mathcal{C}_{x}\right)=f_{0}(\alpha) \\
& 0 \leq \alpha \leq 1 \\
& 0 \leq \mathcal{C}_{x} \leq 1 .
\end{array}
$$

Alternatively, using the equivalent constraint enables us to rewrite the optimization problem in an equivalent form as

$$
\begin{array}{cl}
\max & f_{0}(\alpha) \\
\text { subject to } & \alpha=a\left(\mathcal{C}_{x}\right) \\
& 0 \leq \alpha \leq 1 \\
& 0 \leq \mathcal{C}_{x} \leq 1 .
\end{array}
$$

To solve (21), note that $a\left(\mathcal{C}_{x}\right)$ is monotonically decreasing in $\mathcal{C}_{x}$, see Appendix A for the proof. Furthermore, the objective function, i.e., $f_{0}(\alpha)$, can be shown to be concave with a maximum that occurs at $\alpha=\tilde{\alpha}$, where $\tilde{\alpha}$ is found to be

$$
\tilde{\alpha}=1+\frac{2^{2 R_{\min }}}{p_{\mathrm{s}} \mathcal{G}_{\mathrm{s}}}-\frac{\left(2^{2 R_{\min }}-1\right)}{p_{\mathrm{s}} \mathcal{H}_{\mathrm{s}}} .
$$

Using the decreasing monotonic property of the first constraint in (21) and the concavity characteristics of the objective function, the solution reduces to PGS scheme if $\tilde{\alpha} \geq \alpha_{\mathrm{PGS}}$. On the other hand, if $\tilde{\alpha}<\alpha_{\mathrm{PGS}}$, then the IGS scheme can be adopted and this condition can be simplified using (22) and (10) obtaining

$$
\left(1-\frac{p_{\mathrm{s}} \mathcal{G}_{\mathrm{s}}}{p_{\mathrm{s}} \mathcal{H}_{\mathrm{s}}}\right)\left(2^{2 R_{\min }}-1\right)<-\left(\frac{p_{\mathrm{s}} \mathcal{G}_{\mathrm{s}}+p_{\mathrm{p}} \mathcal{H}_{\mathrm{p}}+1}{2^{R_{\min }}}\right) .
$$

Thus $\mathcal{G}_{\mathrm{s}}>\mathcal{H}_{\mathrm{s}}$ is a necessary condition but not sufficient so that (23) is true. To find the necessary and sufficient conditions, we simplify (23) obtaining

$$
2^{R_{\min }}\left(2^{2 R_{\min }}-1\right)-\beta>0 .
$$

Consider then the cubic equation $x^{3}-x-\beta=0$, the number of positive roots equals 1 according to Descartes rule of signs for the coefficient of the cubic equations [24]. The unique positive root can be found using expressions in [25] which reduces to the condition $R_{\min }>R_{\mathrm{I}, 1}{ }^{4}$. As a result, both

${ }^{4}$ It is worth to mention that for $27 \beta^{2}-4<0$, one can derive that the $R_{\mathrm{I}, 1}$ expression in (15) is a logarithmic function of a sum of two conjugate complex terms with positive real component. 
$\mathcal{G}_{\mathrm{s}}>\mathcal{H}_{\mathrm{s}}$ and $R_{\mathrm{I}, 1}<R_{\min }<R_{0}$ represent the necessary and sufficient conditions so that the IGS based scheme achieves better performance than its PGS counterpart in the feasible overlay cognitive radio system.

As a result from Theorem 3, for $\mathcal{G}_{\mathrm{s}} \leq \mathcal{H}_{\mathrm{s}}$, the optimal signaling scheme is the PGS which coincides with the results in [17]. Is worth to mention that the optimal decoding scheme at the PR is the multiuser decoder, where the primary network detects jointly the interference and the signals which contradicts with the principle that primary network is supposed not to be aware with the interference.

To optimize the statistical signal parameters for IGS in a feasible overlay cognitive radio system with valid conditions of IGS superiority as stated in Theorem 3, we introduce the following Theorem for the optimal adjustment of the IGS scheme.

Theorem 4. In a feasible overlay cognitive radio system with PGS scheme adopted for the primary message, and IGS scheme for the secondary message with $\mathcal{G}_{\mathrm{s}}>\mathcal{H}_{\mathrm{s}}$ and $R_{\min }>$ $R_{\mathrm{I}, 1}$, the optimal $S T$ power distribution between the primary and secondary messages, and the circularity coefficient of the secondary message that maximize the secondary network achievable rate while satisfying a minimum rate requirement $R_{\min }$ to the primary network are expressed as

$$
\left(\alpha_{\mathrm{IGS}}, \mathcal{C}_{x}^{*}\right)= \begin{cases}(\tilde{\alpha}, c(\tilde{\alpha})) & R_{\mathrm{I}, 1}<R_{\min } \leq \min \left(R_{\mathrm{I}, 2}, R_{\mathrm{I}, 3}\right) \\ (a(1), 1) & R_{\mathrm{I}, 2}<R_{\min } \leq R_{\mathrm{I}, 3}, R_{\mathrm{I}, 3}>R_{\mathrm{I}, 2} \\ (0, c(0)) & R_{\mathrm{I}, 3}<R_{\min } \leq R_{\mathrm{I}, 4}, R_{\mathrm{I}, 4}>R_{\mathrm{I}, 3} \\ (a(1), 1) & \max \left(R_{\mathrm{I}, 3}, R_{\mathrm{I}, 4}\right) \leq R_{\min }<R_{0}\end{cases}
$$

where $c(x)$ is defined as

$$
\begin{aligned}
c(x)=\frac{p_{\mathrm{s}} \mathcal{G}_{\mathrm{s}}+p_{\mathrm{p}} \mathcal{H}_{\mathrm{p}}+1}{(1-x) p_{\mathrm{s}} \mathcal{G}_{\mathrm{s}} \sqrt{2^{2 R_{\min }}-1}} \times \\
\sqrt{\frac{\left((1-x) p_{\mathrm{s}} \mathcal{G}_{\mathrm{s}}+1\right)^{2}}{\left(p_{\mathrm{s}} \mathcal{G}_{\mathrm{s}}+p_{\mathrm{p}} \mathcal{H}_{\mathrm{p}}+1\right)^{2}} 2^{2 R_{\min }}-1}
\end{aligned}
$$

and the rate limits are defined as

$$
\begin{gathered}
R_{\mathrm{I}, 2}=\frac{1}{2} \log _{2}\left[\left(\frac{p_{\mathrm{s}} \mathcal{G}_{\mathrm{s}}}{4\left(p_{\mathrm{s}} \mathcal{G}_{\mathrm{s}}-p_{\mathrm{s}} \mathcal{H}_{\mathrm{s}}\right)}\right) \times\right. \\
\left(1+\sqrt{\left.1+8\left(\frac{p_{\mathrm{s}} \mathcal{G}_{\mathrm{s}}}{p_{\mathrm{s}} \mathcal{H}_{\mathrm{s}}}-1\right)\left(\frac{p_{\mathrm{s}} \mathcal{G}_{\mathrm{s}}}{p_{\mathrm{s}} \mathcal{H}_{\mathrm{s}}}+\left(p_{\mathrm{s}} \mathcal{G}_{\mathrm{s}}+p_{\mathrm{p}} \mathcal{H}_{\mathrm{p}}+1\right)^{2}\right)\right)}\right] \\
R_{\mathrm{I}, 3}=\frac{1}{2} \log _{2}\left(\frac{p_{\mathrm{s}} \mathcal{G}_{\mathrm{s}}\left(1+p_{\mathrm{s}} \mathcal{H}_{\mathrm{s}}\right)}{p_{\mathrm{s}} \mathcal{G}_{\mathrm{s}}-p_{\mathrm{s}} \mathcal{H}_{\mathrm{s}}}\right) \\
R_{\mathrm{I}, 4}=\frac{1}{2} \log _{2}\left(\left(1+p_{\mathrm{p}} H_{\mathrm{p}}\right)\left(1+\frac{p_{\mathrm{p}} H_{\mathrm{p}}}{2 p_{\mathrm{s}} G_{\mathrm{s}}+1}\right)\right) .
\end{gathered}
$$

Proof. The IGS optimal solution is found by solving the optimization problem (21) for $\tilde{\alpha}<\alpha_{\mathrm{PGS}}$. Under this assumption we have two cases:

- Case 1: For $0 \leq \tilde{\alpha}<\alpha_{\mathrm{PGS}}$, the IGS solution $\left(\alpha_{\mathrm{IGS}}\right)$ reduces to $\tilde{\alpha}$ as long as the corresponding $\mathcal{C}_{x}$ satisfies its constraint, i.e., $0 \leq \mathcal{C}_{x} \leq 1$, otherwise, $\mathcal{C}_{x}=1$ and $\alpha_{\text {IGS }}$ is assigned the corresponding $\alpha$ from $(19)^{5}$. Thus, the solution of this case is expressed as

$$
\left(\alpha_{\mathrm{IGS}}, C_{x}^{*}\right)= \begin{cases}(\tilde{\alpha}, c(\tilde{\alpha})) & c(\tilde{\alpha})<1 \\ (a(1), 1) & c(\tilde{\alpha}) \geq 1 .\end{cases}
$$

First, it is worthy to note that $c(\alpha)$ is real as shown in Appendix B. Second, one can prove that $0 \leq \tilde{\alpha}<\alpha_{\mathrm{PGS}}$ is equivalent to $R_{\mathrm{I}, 1}<R_{\min } \leq R_{\mathrm{I}, 3}$, see Appendix $\mathrm{C}$ for the proof. In addition, The condition $c(\tilde{\alpha}) \geq 1$ is equivalent to $R_{\min } \geq R_{\mathrm{I}, 2}$, see Appendix $\mathrm{C}$ for the proof. As a result, the solution of (30) reduces to

$$
\left(\alpha_{\mathrm{IGS}}, \mathcal{C}_{x}^{*}\right)= \begin{cases}(\tilde{\alpha}, c(\tilde{\alpha})) & R_{\mathrm{I}, 1}<R_{\min } \leq \min \left(R_{\mathrm{I}, 2}, R_{\mathrm{I}, 3}\right) \\ (a(1), 1) & R_{\mathrm{I}, 2}<R_{\min } \leq R_{\mathrm{I}, 3}, R_{\mathrm{I}, 3}>R_{\mathrm{I}, 2}\end{cases}
$$

- Case 2: For $\tilde{\alpha} \leq 0$, the solution reduces to the minimum feasible $\alpha$. Since the first constraint in (21) is monotonically decreasing in $\mathcal{C}_{x}$, the optimal solution of $\alpha$ is the corresponding value to $\mathcal{C}_{x}=1$ unless it is positive, otherwise $\alpha=0$ and $\mathcal{C}_{x}$ is assigned the corresponding value. The aforementioned solution is written as

$$
\left(\alpha_{\mathrm{IGS}}, \mathcal{C}_{x}^{*}\right)= \begin{cases}(a(1), 1) & a(1)>0 \\ (0, c(0)) & a(1) \leq 0\end{cases}
$$

where $a(1)>0$ reduces to $R_{\min } \leq R_{\mathrm{I}, 4}$, which permits to write the solution in the following form ${ }^{6}$

$$
\left(\alpha_{\mathrm{IGS}}, \mathcal{C}_{x}^{*}\right)=\left\{\begin{array}{cc}
(a(1), 1) & R_{\min } \geq \max \left(R_{\mathrm{I}, 3}, R_{\mathrm{I}, 4}\right) \\
(0, c(0)) & R_{\mathrm{I}, 4} \geq R_{\min } \geq R_{\mathrm{I}, 3}, R_{\mathrm{I}, 4}>R_{\mathrm{I}, 3}
\end{array}\right.
$$

From (30), it is interesting to note that when the network is operating at $R_{\min } \leq R_{1}$, the direct link of the primary network is strong enough even with the existence of interference and does not require any assistant from ST.

According to Theorem 1, maximally improper solution is adopted at specific high rates that are greater than some rate thresholds to relieve the ST interference on the PR received signal.

\section{Overlay Cognitive Radio System Design with PARTIAL-CSI}

Throughout the previous section, the overlay cognitive radio system is designed assuming the availability of CSI at the ST side. Such an assumption is reasonable if the primary network has its CSI at the transmitter side and shares it with the ST through the backhaul connection. In this section, we assume the PT does not have access to its link CSI, while the PR estimates the CSI of the direct primary link for the detection purpose. Therefore, the primary network operates at a fixed rate according to the average CSI of the primary link. As such, the primary network QoS is measured in terms of the outage probability $P_{\text {out }}$ of a target rate $R_{\mathrm{t}}$. The link from the ST to the PR is assumed to be known as a result of

\footnotetext{
${ }^{5}$ This result is based on the decreasing characteristics of $a\left(\mathcal{C}_{x}\right)$ proved in Appendix A

${ }^{6}$ See Appendix D for the proof.
} 
the cooperation between both networks to deliver the primary message. Furthermore, as a result of sharing the primary message with the ST, the ST can be aware with the PT message structure including data and pilots, which helps the SR to estimate the PT to SR channel. In the following, we derive first the outage probability of the primary network in the presence of overlay spectrum sharing. Then, we optimize both the primary and the secondary signals transmitted from the ST to maximize the secondary network achievable rate while satisfying a maximum outage threshold for the target rate.

\section{A. Outage Probability Performance of the Primary Network}

In the following, we derive the outage probability of the primary transmission where overlay cognitive radio uses IGS scheme for the secondary transmission. Then the outage probability of the system when the overlay cognitive radio system uses PGS scheme can be simply obtained by imposing $\mathcal{C}_{x}=0$. The outage probability of the primary transmission of the PGS based system is defined as

$$
P_{\text {out }}\left(\alpha, \mathcal{C}_{x}, R_{\mathrm{t}}\right)=\operatorname{Pr}\left(R_{\mathrm{p}}\left(\alpha, \mathcal{C}_{x}\right)<R_{\mathrm{t}}\right) .
$$

After some mathematical simplifications, we obtain the following equivalent expression

$$
P_{\text {out }}\left(\alpha, \mathcal{C}_{x}, R_{\mathrm{t}}\right)=\operatorname{Pr}\left(q\left(\mathcal{H}_{\mathrm{p}}\right)<0\right),
$$

where $q\left(\mathcal{H}_{\mathrm{p}}\right)$ is found to be

$$
q\left(\mathcal{H}_{\mathrm{p}}\right)=\mathcal{H}_{\mathrm{p}}^{2}+\frac{2}{p_{\mathrm{p}}}\left(1+p_{\mathrm{s}} \mathcal{G}_{\mathrm{s}}\right) \mathcal{H}_{\mathrm{p}}-\frac{1}{p_{\mathrm{p}}^{2}} t\left(\alpha, \mathcal{C}_{x}, R_{\mathrm{t}}\right),
$$

and $t\left(\alpha, \mathcal{C}_{x}, R_{\mathrm{t}}\right)$ is defined as

$$
\begin{array}{r}
t\left(\alpha, \mathcal{C}_{x}, R_{\mathrm{t}}\right)=\left(2^{2 R_{\mathrm{t}}}-1\right)\left[(1-\alpha)^{2}\left(1-\mathcal{C}_{x}^{2}\right)\left(p_{\mathrm{s}} \mathcal{G}_{\mathrm{s}}\right)^{2}\right. \\
\left.\quad+2(1-\alpha) p_{\mathrm{s}} \mathcal{G}_{\mathrm{s}}+1\right]-\alpha p_{\mathrm{s}} \mathcal{G}_{\mathrm{s}}\left(2+(2-\alpha) p_{\mathrm{s}} \mathcal{G}_{\mathrm{s}}\right) .
\end{array}
$$

From (36), we find that $q\left(\mathcal{H}_{\mathrm{p}}\right) \geq 0$ if $t\left(\alpha, \mathcal{C}_{x}, R_{\mathrm{t}}\right) \leq 0$, which reduces to a zero outage scenario. On the other hand, if $t\left(\alpha, \mathcal{C}_{x}, R_{\mathrm{t}}\right)>0$, then $q\left(\mathcal{H}_{\mathrm{p}}\right)$ has one positive and one negative roots. In this case and under the assumption of Rayleigh fading direct transmission channel with average channel-to-noise ratio $\overline{\mathcal{H}}_{\mathrm{p}}$, the outage probability of the primary transmission is expressed as

$$
\begin{aligned}
& P_{\text {out }}\left(\alpha, \mathcal{C}_{x}, R_{\mathrm{t}}\right)=1 \\
& -\exp \left(-\left(\frac{1+p_{\mathrm{s}} \mathcal{G}_{\mathrm{s}}}{p_{\mathrm{p}} \overline{\mathcal{H}}_{\mathrm{p}}}\right)\left(\sqrt{1+\frac{t\left(\alpha, \mathcal{C}_{x}, R_{\mathrm{t}}\right)}{\left(1+p_{\mathrm{s}} \mathcal{G}_{\mathrm{s}}\right)^{2}}}-1\right)\right) .
\end{aligned}
$$

As a result, the outage probability of the primary transmission is expressed generally as

$$
P_{\text {out }}\left(\alpha, \mathcal{C}_{x}\right)=\left\{\begin{array}{cl}
P_{\text {out }}\left(\alpha, \mathcal{C}_{x}, R_{\mathrm{t}}\right) & R_{\mathrm{t}}>R_{\mathrm{z}} \\
0 & R_{\mathrm{t}} \leq R_{\mathrm{z}}
\end{array},\right.
$$

where the condition $R_{\mathrm{t}} \leq R_{\mathrm{z}}$ is an equivalent condition to $t\left(\alpha, \mathcal{C}_{x}, R_{\mathrm{t}}\right) \leq 0$, which can be found after simple manipulations, with $R_{\mathrm{z}}$ is defined as

$$
R_{\mathrm{z}}=\frac{1}{2} \log _{2}\left(1+\frac{\left(\alpha p_{\mathrm{s}} \mathcal{G}_{\mathrm{s}}\right)\left(2+(2-\alpha) p_{\mathrm{s}} \mathcal{G}_{\mathrm{s}}\right)}{(1-\alpha)^{2}\left(1-\mathcal{C}_{x}^{2}\right)\left(p_{\mathrm{s}} \mathcal{G}_{\mathrm{s}}\right)^{2}+2(1-\alpha) p_{\mathrm{s}} \mathcal{G}_{\mathrm{s}}+1}\right) .
$$

From (4) and (40), we observe that $R_{\mathrm{z}}$ indicates that the ST channel to the PR is strong and can achieve the primary network target rate besides possible existence of secondary transmission and without considering the PT direct link.

\section{B. Proper Gaussian Signaling with Partial CSI based Design}

The PGS based design using partial CSI is achieved by solving the following optimization problem to distribute the ST power between the primary and secondary messages

$$
\begin{array}{cl}
\max & R_{\mathrm{s}}(\alpha, 0) \\
\text { subject to } & P_{\text {out,p }}\left(\alpha, 0, R_{\mathrm{t}}\right) \leq P_{\text {out }, \text { th }} \\
& 0 \leq \alpha \leq 1 .
\end{array}
$$

First, we analyze the problem (41) to find the feasibility condition of overlay spectrum sharing with partial CSI based on the following theorem.

Theorem 5. Assume a primary network that operates at a target rate $R_{\mathrm{t}}$ and maximum outage probability $P_{\mathrm{out}, \mathrm{th}}$, and assume the availability of its link average CSI and the secondary links instantaneous CSI at the ST side. Then, the ST delivers its data to the $S R$ and maintains the primary network QoS if and only if $R_{\mathrm{t}}<\hat{R}_{0}$, where $\hat{R}_{0}$ is given by

$$
\hat{R}_{0}=\log _{2}\left(1+p_{\mathrm{s}} \mathcal{G}_{\mathrm{s}}+p_{\mathrm{p}} \overline{\mathcal{H}}_{\mathrm{p}} \log \left(\frac{1}{1-P_{\text {out }, \text { th }}}\right)\right) .
$$

Proof. The maximum target rate of the primary network is achieved with an outage probability of $P_{\text {out,th }}$ when the ST is fully dedicated to the primary network. This rate is computed by equating (38) to $P_{\text {out,th }}$, and setting $\alpha=1$, then find $R_{\mathrm{t}}=$ $\hat{R}_{0}$ after simple manipulations.

According to Theorem 5, if $R_{\mathrm{t}}>\hat{R}_{0}$ no feasible overlay cognitive radio exists, which means that both the primary link and secondary link to the PR are not reliable and fails to achieve the required QoS of the primary network. On the other hand, when the feasibility condition is valid, the ST uses a portion of its power budget to send the primary message and the rest to send the secondary message while satisfying the primary network QoS. Moreover, this feasibility condition applies regardless of the secondary message signaling scheme whether it is PGS or IGS similar to the full-CSI based design. The next result states the ST signal design parameters that maximize the secondary network achievable rate while satisfying the primary network QoS.

Theorem 6. Assume a primary network that operates at a target rate $R_{\mathrm{t}}$ and maximum outage probability $P_{\mathrm{out}, \mathrm{th}}$, and assume the availability of its link average CSI and the secondary links instantaneous CSI at the ST side. Then, a feasible overlay cognitive radio system with PGS scheme adopted at both the primary and secondary networks is optimally designed by dividing the ST power $p_{\mathrm{s}}$ between the primary message and the secondary message as $\hat{\alpha}_{\mathrm{PGS}} p_{\mathrm{s}}$ and $\left(1-\hat{\alpha}_{\mathrm{PGS}}\right) p_{\mathrm{s}}$, respectively, with $\hat{\alpha}_{\mathrm{PGS}}$ given by

$$
\hat{\alpha}_{\mathrm{PGS}}=\left\{\begin{array}{cc}
\rho_{\mathrm{L}} & \hat{R}_{\mathrm{p}, 1}<R_{\mathrm{t}} \leq \hat{R}_{0} \\
0 & R_{\mathrm{t}} \leq \hat{R}_{\mathrm{p}, 1}
\end{array}\right.
$$


where $\rho_{\mathrm{L}}$ is defined as

$$
\begin{aligned}
& \rho_{\mathrm{L}}=\left(\frac{p_{\mathrm{s}} \mathcal{G}_{\mathrm{s}}+1}{p_{\mathrm{s}} \mathcal{G}_{\mathrm{s}}}\right) \times \\
& \left(1-\frac{1}{2^{R_{\mathrm{t}}}}\left(\left(\frac{p_{\mathrm{p}} \overline{\mathcal{H}}_{\mathrm{p}}}{1+p_{\mathrm{s}} \mathcal{G}_{\mathrm{s}}}\right) \log \left(\frac{1}{1-P_{\text {out }, \text { th }}}\right)+1\right)\right),
\end{aligned}
$$

and $\hat{R}_{\mathrm{p}, 1}$ is expressed as

$$
\hat{R}_{\mathrm{p}, 1}=\log _{2}\left(1+\left(\frac{p_{\mathrm{p}} \overline{\mathcal{H}}_{\mathrm{p}}}{1+p_{\mathrm{s}} \mathcal{G}_{\mathrm{s}}}\right) \log \left(\frac{1}{1-P_{\text {out }, \text { th }}}\right)\right) .
$$

Proof. First the optimization problem in (41) can be simplified obtaining

$$
\begin{gathered}
\min _{\alpha} \quad \alpha \\
\text { subject to } \alpha^{2}-2\left(\frac{p_{\mathrm{s}} \mathcal{G}_{\mathrm{s}}+1}{p_{\mathrm{s}} \mathcal{G}_{\mathrm{s}}}\right) \alpha+\left(\frac{1+p_{\mathrm{s}} \mathcal{G}_{\mathrm{s}}}{p_{\mathrm{s}} \mathcal{G}_{\mathrm{s}}}\right)^{2} \times \\
\left(1-\frac{1}{2^{2 R_{\mathrm{t}}}}\left(\frac{p_{\mathrm{p}} \overline{\mathcal{H}}_{\mathrm{p}}}{1+p_{\mathrm{s}} \mathcal{G}_{\mathrm{s}}} \log \frac{1}{1-P_{\text {out }, \text { th }}}+1\right)^{2}\right) \leq 0 \\
0 \leq \alpha \leq 1 .
\end{gathered}
$$

After finding the roots of the constraint, the optimization problem is simplified to

$$
\begin{array}{cl}
\min _{\alpha} & \alpha \\
\text { subject to } & \rho_{\mathrm{L}} \leq \alpha \leq \rho_{\mathrm{U}} \\
& 0 \leq \alpha \leq 1,
\end{array}
$$

where $\rho_{\mathrm{U}}$ is found to be

$$
\begin{aligned}
\rho_{\mathrm{U}} & =\left(\frac{p_{\mathrm{s}} \mathcal{G}_{\mathrm{s}}+1}{p_{\mathrm{s}} \mathcal{G}_{\mathrm{s}}}\right) \times \\
& \left(1+\frac{1}{2^{R_{\mathrm{t}}}}\left(\left(\frac{p_{\mathrm{p}} \overline{\mathcal{H}}_{\mathrm{p}}}{1+p_{\mathrm{s}} \mathcal{G}_{\mathrm{s}}}\right) \log \left(\frac{1}{1-P_{\text {out }, \text { th }}}\right)+1\right)\right) .
\end{aligned}
$$

One can show easily that $\rho_{\mathrm{U}}>1$, on the other hand, it is not necessary the same apply for $\rho_{\mathrm{L}}$. As such, we have the following three cases

- $\rho_{\mathrm{L}}>1$, which results in an solution. In this case, $\rho_{\mathrm{L}}>1$ is reduced to $R_{\mathrm{t}}>\hat{R}_{0}$, which reflects the infeasible existence of overlay cognitive radio as pointed in Theorem 5.

- $0<\rho_{\mathrm{L}}<1$, the solution reduces to minimum feasible $\alpha$ giving $\hat{\alpha}_{\mathrm{PGS}}=\rho_{\mathrm{L}}$

- $\rho_{\mathrm{L}}<0$, the solution reduces to $\alpha_{\mathrm{PGS}}=0$, which reflect the strong primary transmission link that achieves the required QoS even with full interference load from the secondary link.

Similar to the full-CSI knowledge based design, IGS scheme can be implemented only when $\hat{R}_{\mathrm{p}, 1}<R_{\mathrm{t}} \leq \hat{R}_{0}$, where for $R_{\mathrm{t}}>\hat{R}_{0}$, the overlay spectrum sharing can not exits according to Theorem 5. On the other hand, when $R_{\mathrm{t}} \leq \hat{R}_{0}$, the scenario reduces to transmission over AWGN, which reduces to PGS scheme as the optimal scheme. The optimal IGS design for $\hat{R}_{\mathrm{p}, 1}<R_{\mathrm{t}} \leq \hat{R}_{0}$ is obtained from the following Theorem.
Theorem 7. Assume a primary network operates at a target rate $R_{\mathrm{t}}$ using PGS scheme and maximum outage probability $P_{\mathrm{out}, \mathrm{th}}$, and assume the availability of its link average CSI and the secondary links instantaneous CSI at the ST side. Then, adopting IGS scheme at the secondary network of a feasible overlay cognitive radio system improves the secondary network achievable rate compared with PGS based scheme if and only if:

1) $\mathcal{G}_{\mathrm{s}}>\mathcal{H}_{\mathrm{s}}$

2) $\hat{R}_{\mathrm{I}, 1}<R_{\min }<\hat{R}_{0}$, where

$$
\begin{array}{r}
\hat{R}_{\mathrm{I}, 1}=\log _{2}\left(\frac{(2 / 3)^{1 / 3}}{\left(9 \hat{\beta}+\sqrt{3} \sqrt{-4+27 \hat{\beta}^{2}}\right)^{1 / 3}}\right. \\
\left.+\frac{\left(9 \hat{\beta}+\sqrt{3} \sqrt{-4+27 \hat{\beta}^{2}}\right)^{1 / 3}}{2^{1 / 3} 3^{2 / 3}}\right)
\end{array}
$$

and $\hat{\beta}=\frac{p_{\mathrm{s}} \mathcal{H}_{\mathrm{s}}\left(1+p_{\mathrm{s}} \mathcal{G}_{\mathrm{s}}-p_{\mathrm{p}} \overline{\mathcal{H}}_{\mathrm{p}} \log \left(1-P_{\mathrm{out}, \mathrm{th}}\right)\right)}{\left(p_{\mathrm{s}} \mathcal{G}_{\mathrm{s}}-p_{\mathrm{s}} \mathcal{H}_{\mathrm{s}}\right)}$.

Proof. First, consider the optimization problem that tunes $\alpha, \mathcal{C}_{x}$ to maximize $R_{\mathrm{s}}$ while achieving a primary link target rate with a maximum outage $P_{\text {out,th }}$.

$$
\begin{array}{cl}
\max _{\alpha, \mathcal{C}_{x}} & R_{\mathrm{s}}\left(\alpha, \mathcal{C}_{x}\right) \\
\text { subject to } & P_{\text {out,p }}\left(\alpha, \mathcal{C}_{x}\right) \leq P_{\text {out }, \text { th }} \\
& 0 \leq \alpha \leq 1 \\
& 0 \leq \mathcal{C}_{x} \leq 1
\end{array}
$$

then, rewrite it using the logarithmic characteristics of the objective function and after simplifying the constraints obtaining the following equivalent optimization function

$$
\begin{array}{cl}
\max _{\alpha, \mathcal{C}_{x}} & 2(1-\alpha)+\left(1-\mathcal{C}_{x}^{2}\right)(1-\alpha)^{2} p_{\mathrm{s}} \mathcal{H}_{\mathrm{s}} \\
\text { subject to } & \mathcal{C}_{x}^{2} \geq f_{\mathrm{p}}(\alpha) \\
& 0 \leq \alpha \leq 1 \\
& 0 \leq \mathcal{C}_{x} \leq 1
\end{array}
$$

where $f_{\mathrm{p}}(\alpha)$ is defined as

$$
\begin{aligned}
f_{\mathrm{p}}(\alpha)= & 1-\left(\frac{1}{2^{2 R_{\mathrm{t}}}-1}\right)\left(\frac{1+p_{\mathrm{s}} \mathcal{G}_{\mathrm{s}}}{(1-\alpha) p_{\mathrm{s}} \mathcal{G}_{\mathrm{s}}}\right)^{2} \times \\
& {\left[\left(\left(\frac{p_{\mathrm{p}} \overline{\mathcal{H}}_{\mathrm{p}}}{1+p_{\mathrm{s}} \mathcal{G}_{\mathrm{s}}}\right) \log \left(\frac{1}{1-P_{\mathrm{out}, \mathrm{th}}}\right)+1\right)^{2}-1\right] } \\
- & \frac{2 \alpha}{(1-\alpha)^{2} p_{\mathrm{s}} \mathcal{G}_{\mathrm{s}}}\left(\frac{2^{2 R_{\mathrm{t}}}+p_{\mathrm{s}} \mathcal{G}_{\mathrm{s}}}{2^{2 R_{\mathrm{t}}}-1}\right)+\frac{\alpha^{2}}{(1-\alpha)^{2}\left(2^{2 R_{\mathrm{t}}}-1\right)} \\
+ & \frac{1+2 p_{\mathrm{s}} \mathcal{G}_{\mathrm{s}}}{\left(p_{\mathrm{s}} \mathcal{G}_{\mathrm{s}}\right)^{2}(1-\alpha)^{2}} .
\end{aligned}
$$


Since the objective function is monotonically decreasing in $\alpha$ and $\mathcal{C}_{x}$, then the optimization problem solution exists at $\mathcal{C}_{x}^{2}=f_{\mathrm{p}}(\alpha)$, which enables us to rewrite equivalently it as

$$
\begin{array}{ll}
\max _{\alpha, \mathcal{C}_{x}} & 2(1-\alpha)+\left(1-f_{\mathrm{p}}(\alpha)\right)(1-\alpha)^{2} p_{\mathrm{s}} \mathcal{H}_{\mathrm{s}} \\
\text { subject to } & 0 \leq f_{\mathrm{p}}(\alpha) \leq 1 \\
& 0 \leq \alpha \leq 1
\end{array}
$$

After further simplification of the objective function, studying the roots of $f_{\mathrm{p}}(\alpha)=0$ and $f_{\mathrm{p}}(\alpha)-1=0$, the optimization problem can be written in its simplest form as

$$
\begin{array}{cl}
\max _{\alpha, \mathcal{C}_{x}} & g(\alpha) \\
\text { subject to } & \alpha_{\mathrm{L}}<\alpha<\alpha_{\mathrm{U}} \\
& 0 \leq \alpha \leq 1
\end{array}
$$

where $g(\alpha)$ is found to be

$$
\begin{gathered}
g(\alpha)=-\frac{\alpha^{2} p_{\mathrm{s}} \mathcal{H}_{\mathrm{s}}}{\left(2^{R_{\mathrm{th}}}-1\right)}+2 \alpha\left(\frac{p_{\mathrm{s}} \mathcal{H}_{\mathrm{s}}}{p_{\mathrm{s}} \mathcal{G}_{\mathrm{s}}}\left(\frac{2^{R_{\mathrm{th}}}+p_{\mathrm{s}} \mathcal{G}_{\mathrm{s}}}{2^{R_{\mathrm{th}}}-1}\right)-1\right) \\
+2-\frac{\left(1+2 p_{\mathrm{s}} \mathcal{G}_{\mathrm{s}}\right) p_{\mathrm{s}} \mathcal{H}_{\mathrm{s}}}{\left(p_{\mathrm{s}} \mathcal{G}_{\mathrm{s}}\right)^{2}}+\left(\frac{p_{\mathrm{s}} \mathcal{H}_{\mathrm{s}}}{2^{2 R_{\mathrm{t}}}-1}\right)\left(\frac{1+p_{\mathrm{s}} \mathcal{G}_{\mathrm{s}}}{p_{\mathrm{s}} \mathcal{G}_{\mathrm{s}}}\right)^{2} \times \\
{\left[\left(\left(\frac{p_{\mathrm{p}} \overline{\mathcal{H}}_{\mathrm{p}}}{1+p_{\mathrm{s}} \mathcal{G}_{\mathrm{s}}}\right) \log \left(\frac{1}{1-P_{\text {out }, \mathrm{th}}}\right)+1\right)^{2}-1\right],}
\end{gathered}
$$

$\alpha_{\mathrm{U}}=\rho_{\mathrm{L}}$ and $\alpha_{\mathrm{L}}$ is found to be

$\alpha_{\mathrm{L}}=\left(\frac{2^{2 R_{\mathrm{t}}}}{p_{\mathrm{s}} \mathcal{G}_{\mathrm{s}}}+1\right)-$

$\sqrt{\left(\frac{2^{R_{\mathrm{t}}}}{p_{\mathrm{s}} \mathcal{G}_{\mathrm{s}}}\right)^{2}\left(2^{2 R_{\mathrm{t}}}-1\right)+\left(\frac{1+p_{\mathrm{s}} \mathcal{G}_{\mathrm{s}}}{p_{\mathrm{s}} \mathcal{G}_{\mathrm{s}}}\right)^{2}\left(\frac{p_{\mathrm{p}} \overline{\mathcal{H}}_{\mathrm{p}}}{1+p_{\mathrm{s}} \mathcal{G}_{\mathrm{s}}} \log \left(\frac{1}{1-P_{\text {out }, \text { th }}}\right)+1\right)^{2}}$.

One can prove that $g(\alpha)$ is a concave function with a peak $\widetilde{\alpha}$ that is expressed as

$$
\widetilde{\alpha}=1+\frac{2^{2 R_{\mathrm{t}}}}{p_{\mathrm{s}} \mathcal{G}_{\mathrm{s}}}-\left(\frac{2^{2 R_{\mathrm{t}}}-1}{p_{\mathrm{s}} \mathcal{H}_{\mathrm{s}}}\right) .
$$

As a result, if $\widetilde{\alpha} \leq \alpha_{\mathrm{U}}=\hat{\alpha}_{\mathrm{PGS}}$, then the solution reduces to PGS solution, otherwise, the IGS scheme is adopted. The later case reduces to the following condition

$$
\begin{aligned}
\left(2^{2 R_{\mathrm{t}}}-1\right) & \left(\frac{1}{p_{\mathrm{s}} \mathcal{G}_{\mathrm{s}}}-\frac{1}{p_{\mathrm{s}} \mathcal{H}_{\mathrm{s}}}\right)<-\frac{1}{2^{R_{\mathrm{t}}}} \times \\
& {\left[\left(\frac{p_{\mathrm{p}} \overline{\mathcal{H}}_{\mathrm{p}}}{p_{\mathrm{s}} \mathcal{G}_{\mathrm{s}}}\right) \log \left(\frac{1}{1-P_{\text {out }, \text { th }}}\right)+\frac{1}{p_{\mathrm{s}} \mathcal{G}_{\mathrm{s}}}+1\right], }
\end{aligned}
$$

which is true if $\mathcal{G}_{\mathrm{s}}>\mathcal{H}_{\mathrm{s}}$. Moreover, the condition can be simplified to $2^{R_{\mathrm{t}}}\left(2^{2 R_{\mathrm{t}}}-1\right)-\hat{\beta}>0$. Similar to the perfect CSI analysisthe IGS usage condition in (58) reduces to

- $\mathcal{G}_{\mathrm{s}}>\mathcal{H}_{\mathrm{s}}$

- $R_{\mathrm{t}}>\hat{R}_{\mathrm{I}, 1}$, where $\hat{R}_{\mathrm{I}, 1}$ has the same expression of (15) with $\beta$ replaced by $\hat{\beta}$.

Similar to the full-CSI IGS based design, the partial-CSI IGS based design achieves benefits for high rate values of feasible overlay cognitive radio. Under the assumption of beneficial IGS based design on overlay cognitive radio systems, the statistical signal parameters can be optimally designed using the following Theorem.

Theorem 8. Assume a primary network operates at a target rate $R_{\mathrm{t}}$ using $P G S$ scheme and maximum outage probability $P_{\mathrm{out}, \mathrm{th}}$, and assume the availability of its link average CSI and the secondary links instantaneous CSI at the ST side. Suppose the secondary network adopts IGS scheme for the secondary message with $\mathcal{G}_{\mathrm{s}}>\mathcal{H}_{\mathrm{s}}$ and $\hat{R}_{0}>R_{\mathrm{t}}>\hat{R}_{\mathrm{I}, 1}$. Then, the optimal $\alpha$ and $\mathcal{C}_{x}$ that maximize $R_{\mathrm{s}}\left(\alpha, \mathcal{C}_{x}\right)$ while satisfying the primary network $Q o S$ are expressed as

$$
\left(\hat{\alpha}_{\mathrm{IGS}}, \hat{\mathcal{C}}_{x}^{*}\right)=\left\{\begin{array}{cc}
\left(\bar{\alpha}, \sqrt{f_{\mathrm{p}}(\widetilde{\alpha})}\right) & R_{\mathrm{I}, 3}<R_{\mathrm{t}}<R_{\mathrm{I}, 2}, R_{\mathrm{I}, 2}>R_{\mathrm{I}, 3} \\
\left(\widetilde{\alpha}, \sqrt{f_{\mathrm{p}}(\widetilde{\alpha})}\right) & R_{\mathrm{t}}<\min \left(R_{\mathrm{I}, 2}, R_{\mathrm{I}, 3}, R_{\mathrm{I}, 4}\right) \\
\left(0, \sqrt{f_{\mathrm{p}}(0)}\right) & R_{\mathrm{I}, 4}<R_{\mathrm{t}}<\min \left(R_{\mathrm{I}, 2}, R_{\mathrm{I}, 3}\right) \\
\left(\alpha_{\mathrm{L}}, 1\right) & R_{\mathrm{t}}>\max \left(R_{\mathrm{I}, 2}, R_{\mathrm{I}, 3}\right) \\
\left(0, \sqrt{f_{\mathrm{p}}(0)}\right) & R_{\mathrm{I}, 2}<R_{\mathrm{t}}<R_{\mathrm{I}, 3}, R_{\mathrm{I}, 3}>R_{\mathrm{I}, 2}
\end{array}\right.
$$

where $R_{\mathrm{I}, 2}, R_{\mathrm{I}, 3}$ and $R_{\mathrm{I}, 4}$ are defined, respectively, as

$$
\begin{aligned}
R_{\mathrm{I}, 2}=\frac{1}{2} \log _{2}\left(\frac{2\left(p_{\mathrm{s}} \mathcal{G}_{\mathrm{s}}\right)^{2}-\left(p_{\mathrm{s}} \mathcal{H}_{\mathrm{s}}\right)^{2}}{2\left(\left(p_{\mathrm{s}} \mathcal{G}_{\mathrm{s}}\right)^{2}-\left(p_{\mathrm{s}} \mathcal{H}_{\mathrm{s}}\right)^{2}\right)} \times\right. \\
\left.\left(1+\sqrt{1+4 \Lambda \frac{\left(p_{\mathrm{s}} \mathcal{G}_{\mathrm{s}}\right)^{2}-\left(p_{\mathrm{s}} \mathcal{H}_{\mathrm{s}}\right)^{2}}{\left(2\left(p_{\mathrm{s}} \mathcal{G}_{\mathrm{s}}\right)^{2}-\left(p_{\mathrm{s}} \mathcal{H}_{\mathrm{s}}\right)^{2}\right)^{2}}}\right)\right),
\end{aligned}
$$

with $\Lambda$ is defined as

$$
\begin{gathered}
\Lambda=\left(p_{\mathrm{s}} \mathcal{H}_{\mathrm{s}}\right)^{2}\left(1+p_{\mathrm{s}} \mathcal{G}_{\mathrm{s}}\right)^{2}\left(\frac{p_{\mathrm{p}} \overline{\mathcal{H}}_{\mathrm{p}}}{1+p_{\mathrm{s}} \mathcal{G}_{\mathrm{s}}} \log \left(\frac{1}{1-P_{\mathrm{out}, \mathrm{th}}}\right)+1\right)^{2} \\
-\left(p_{\mathrm{s}} \mathcal{G}_{\mathrm{s}}\right)^{2} \\
R_{\mathrm{I}, 3}=\frac{1}{2} \log _{2}\left(\frac{\left(1+p_{\mathrm{s}} \mathcal{G}_{\mathrm{s}}\right)^{2}}{2 p_{\mathrm{s}} \mathcal{G}_{\mathrm{s}}+1}\left(\frac{p_{\mathrm{p}} \overline{\mathcal{H}}_{\mathrm{p}}}{1+p_{\mathrm{s}} \mathcal{G}_{\mathrm{s}}} \log \left(\frac{1}{1-P_{\mathrm{out}, \mathrm{th}}}\right)+1\right)^{2}\right. \\
\left.-\frac{\left(p_{\mathrm{s}} \mathcal{G}_{\mathrm{s}}\right)^{2}}{2 p_{\mathrm{s}} \mathcal{G}_{\mathrm{s}}+1}\right) \\
R_{\mathrm{I}, 4}=\frac{1}{2} \log _{2}\left(\frac{\left(p_{\mathrm{s}} \mathcal{H}_{\mathrm{s}}+1\right) p_{\mathrm{s}} \mathcal{G}_{\mathrm{s}}}{p_{\mathrm{s}} \mathcal{G}_{\mathrm{s}}-p_{\mathrm{s}} \mathcal{H}_{\mathrm{s}}}\right)
\end{gathered}
$$

Proof. Consider the optimization problem, we find that we have the following possible cases

- $\alpha_{\mathrm{L}}<\breve{\alpha}<\alpha_{\mathrm{U}}, \quad \alpha_{\mathrm{L}}>0$, which reduces the solution to $\left(\widetilde{\alpha}, \sqrt{f_{\mathrm{p}}(\breve{\alpha})}\right)$

- $\alpha_{\mathrm{L}}<\widetilde{\alpha}<\alpha_{\mathrm{U}}, \alpha_{\mathrm{L}}<0, \quad \widetilde{\alpha}>0$, which reduces also to $\left(\widetilde{\alpha}, \sqrt{f_{\mathrm{p}}(\breve{\alpha})}\right)$

- $\alpha_{\mathrm{L}}<\breve{\alpha}<\alpha_{\mathrm{U}}, \quad \alpha_{\mathrm{L}}<0, \quad \bar{\alpha}<0$, which reduces to $\left(0, \sqrt{f_{\mathrm{p}}(0)}\right)$

- $\alpha<\alpha_{\mathrm{L}}, \alpha_{\mathrm{L}}>0$, which reduces to $\left(\alpha_{\mathrm{L}}, 1\right)$

- $\bar{\alpha}<\alpha_{\mathrm{L}}, \alpha_{\mathrm{L}}<0$, which reduces to $\left(0, \sqrt{f_{\mathrm{p}}(0)}\right)$. 
Each case can be equivalently written in terms of $R_{\mathrm{r}}$ and other rate threshold values in a similar way done with the full-CSI based design ${ }^{7}$.

\section{NumERICAL RESUlts}

In this section, we evaluate numerically the rate performance gain that is reaped by adopting IGS scheme compared with PGS scheme in the overlay cognitive radio using full CSI and partial CSI based design. Throughout the following numerical results, we assume the following system parameters, unless otherwise specified, $p_{\mathrm{p}}=p_{\mathrm{s}}=1, R_{\min }=R_{\mathrm{t}}=4 \mathrm{bit} / \mathrm{s} / \mathrm{Hz}$, $\overline{\mathcal{H}}_{\mathrm{p}}=\mathbb{E}\left[\mathcal{H}_{\mathrm{p}}\right]=15 \mathrm{~dB}, \overline{\mathcal{G}}_{\mathrm{s}}=\mathbb{E}\left[\mathcal{G}_{\mathrm{s}}\right]=20 \mathrm{~dB}, P_{\text {out }, \text { th }}=10^{-2}$ and $\overline{\mathcal{H}}_{\mathrm{s}}=\mathbb{E}\left[\mathcal{H}_{\mathrm{s}}\right]=10 \mathrm{~dB}$.

Firstly, we study the impact of $\mathcal{G}_{\mathrm{s}}$ on the benefit of IGS scheme over PGS scheme using the full-CSI based design. To this end, we compare the average $R_{\mathrm{S}}$ of the PGS and the IGS based schemes versus $\mathcal{G}_{\mathrm{s}}$ in Fig. 2. Furthermore, we plot in the same figure the average optimal parameters, $\mathcal{C}_{x}^{*}$,

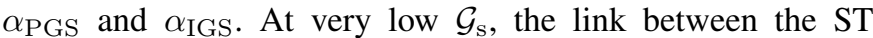
and the PR is weak so that the interference may be neglected with respect to the primary link signal strength. As a result, both links operate without interference effect. In this case, the IGS solution reduces to the PGS solution. As $\mathcal{G}_{\mathrm{s}}$ increases, the average $R_{\mathrm{S}}$ for the PGS based scheme degrades as a result of increasing the interference on the PR, which requires increasing $\alpha$ to satisfy the primary network QoS. On the other hand, the benefit of employing IGS scheme starts to appear where $\mathcal{C}_{x}^{*}$ increases to reduce the interference effect on the SR. Furthermore, this interference relief allows decreasing $\alpha$, which creates a room to increase the secondary message power. Although increasing $\mathcal{C}_{x}^{*}$ and decreasing $\alpha$ have contradicting effect on $R_{\mathrm{s}}$, optimizing these parameters together using the proposed optimal solution guarantees improving $R_{\mathrm{S}}$ of the IGS based scheme compared with the PGS one. This improvement appears as a reduction of the $R_{\mathrm{S}}$ degradation in the low to medium range of $\mathcal{G}_{\mathrm{s}}$ and converts to an improvement at medium to high values of $\mathcal{G}_{\mathrm{s}}$. At very high $\mathcal{G}_{\mathrm{s}}, R_{\mathrm{s}}$ becomes constant because both $\mathcal{C}_{x}^{*}$ and $\left(1-\alpha_{\text {IGS }}\right)$ go to one.

Secondly, we investigate the effect of $R_{\min }$ and $\overline{\mathcal{H}}_{\mathrm{p}}$ on the average rate performance gain of the IGS based scheme over the PGS one. For this purpose, we plot the average $R_{\mathrm{s}}$ for both schemes versus $R_{\text {min }}$ assuming $\overline{\mathcal{H}}_{\mathrm{p}}=10,20,30 \mathrm{~dB}$ in Fig. 3. According to the shown results, we report three observations. The first is that the maximum benefit of employing IGS scheme lies in the middle minimum rate requirement range. On the other hand, at low and high values of $R_{\min }$, the IGS solution reduces approximately to the PGS one. The second observation is that the IGS based scheme benefit exists at low and high $\overline{\mathcal{H}}_{\mathrm{p}}$. The aforementioned observation matches the results of Theorem 3 . The third observation is that the maximum relative performance improvement at specific $R_{\min }$ exists at low $\overline{\mathcal{H}}_{\mathrm{p}}$, for example $50 \%$ improvement can be obtained at $R_{\min }=4$.

Thirdly, under the assumption of partial CSI, we study the impact of $\mathcal{G}_{\mathrm{s}}$ on the benefit of the IGS scheme based design over the PGS one. Figure 4 indicates that the average $R_{\mathrm{S}}$ for

${ }^{7}$ The proof is omitted since it is straightforward and due to space limitations

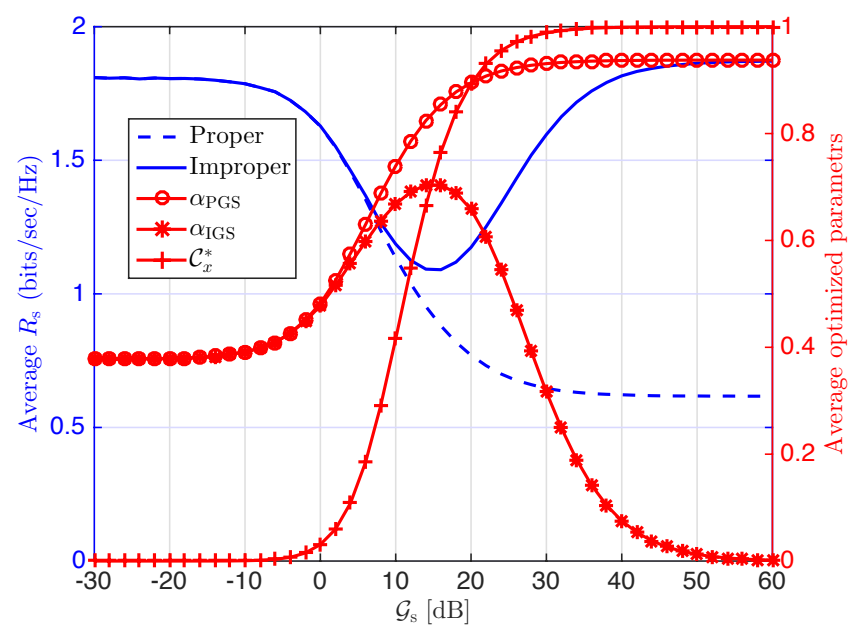

Fig. 2. Average $R_{\mathrm{S}}$ versus $\mathcal{G}_{\mathrm{S}}$ for both PGS and IGS based schemes along with the corresponding average optimized parameters.

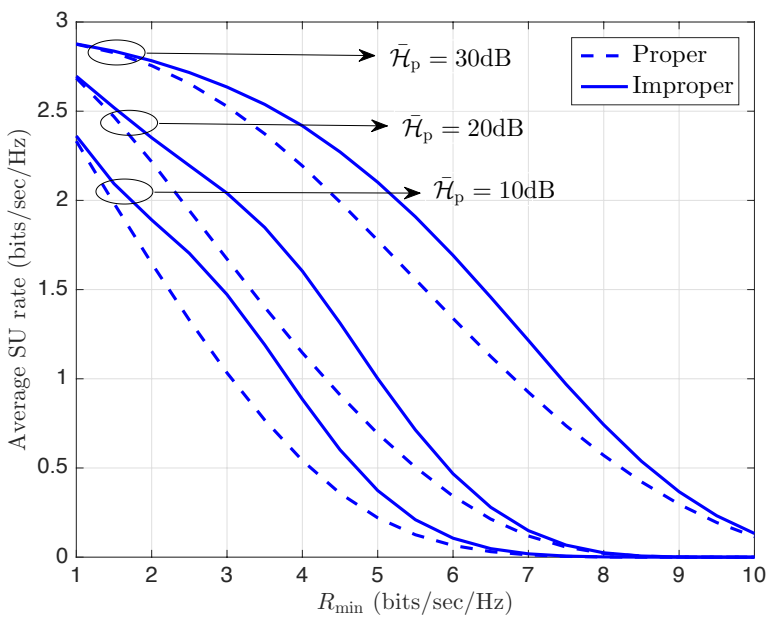

Fig. 3. Average $R_{\mathrm{S}}$ versus $R_{\mathrm{min}}$ for both PGS and IGS based schemes assuming $\overline{\mathcal{H}}_{\mathrm{p}}=10,20,30 \mathrm{~dB}$.

both the PGS and the IGS based schemes performance along with the average values of design variables, i.e., $\alpha_{\mathrm{PGS}}, \alpha_{\mathrm{IGS}}$ and $\mathcal{C}_{x}$. At low average values of $\mathcal{G}_{\mathrm{s}}$ both the PGS and the IGS based schemes tend to fully dedicate the ST power to support the primary message delivery. The observed results is due to the lack of instantaneous information of $\mathcal{H}_{\mathrm{p}}$. As $\overline{\mathcal{G}}_{\mathrm{s}}$ increases, the ST can use partial of its power for the primary message and the rest for the secondary message. Furthermore, different from the full CSI based design at which $\alpha_{\mathrm{PGS}}$ increases with the increase of $\overline{\mathcal{G}}_{\mathrm{s}}$, the situation is reversed. The mean reason of the previously described observation is the uncertainty knowledge of $\mathcal{H}_{\mathrm{p}}$, where it is only available on average in addition to low values of $\mathcal{G}_{\mathrm{s}}$. At large average values of $\mathcal{G}_{\mathrm{s}}$, the ST power assigned mostly to the secondary message and $\mathcal{C}_{\S}$ increases to reduce the interference impact on the $\mathrm{PR}$. As for the relative improvement the IGS based scheme can provide three times improvement of the PGS based scheme.

Fourthly, to emphasize on the effect of partial CSI knowl- 


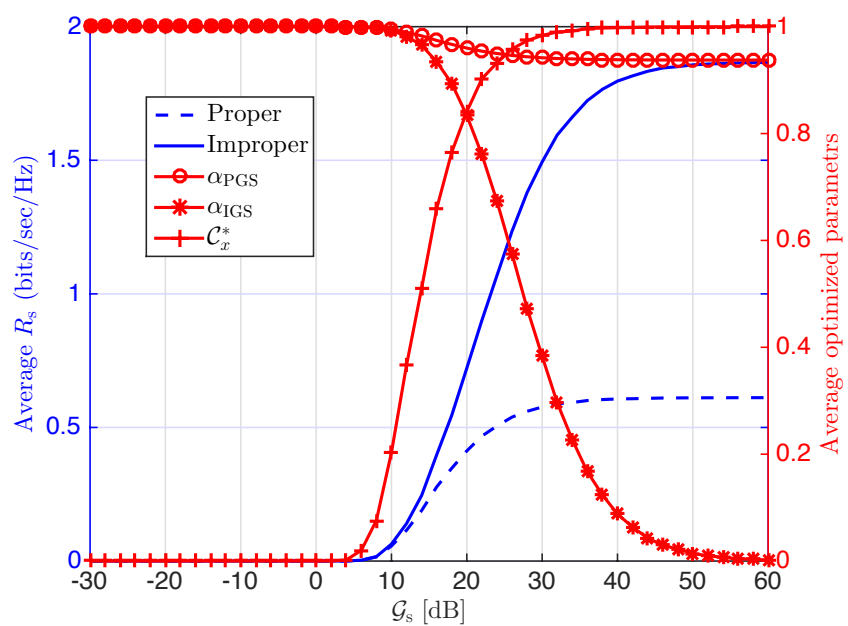

Fig. 4. Average $R_{\mathrm{S}}$ versus $\mathcal{G}_{\mathrm{s}}$ for both PGS and IGS based schemes along with the corresponding average optimized parameters with partial CSI.

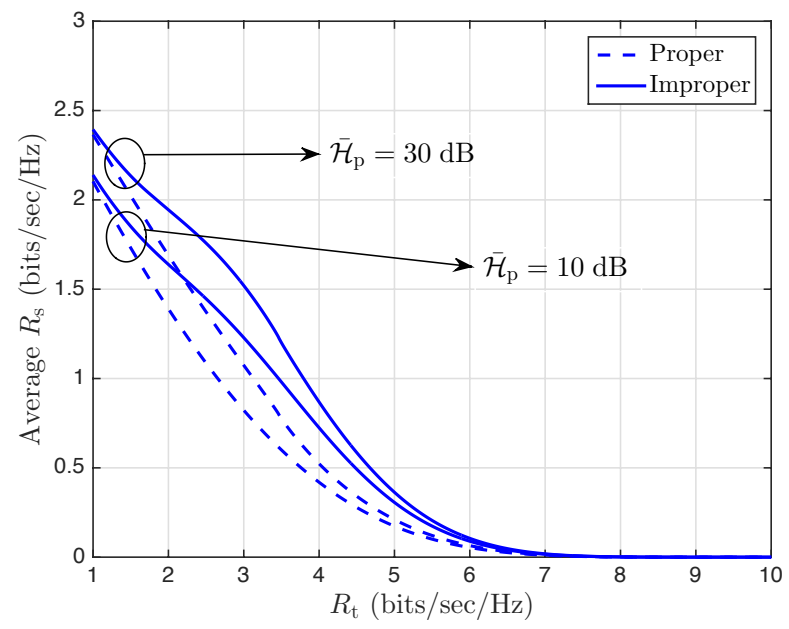

Fig. 5. Average $R_{\mathrm{S}}$ versus $R_{\mathrm{min}}$ for both PGS and IGS based schemes assuming $\overline{\mathcal{H}}_{\mathrm{p}}=10$, and $30 \mathrm{~dB}$ using partial CSI.

edge design based on the secondary network average achievable rate, we plot it versus the target rate for $\overline{\mathcal{H}}_{\mathrm{p}}=10$, and $20 \mathrm{~dB}$ in Fig. 5. With the comparison of these results and the full CSI based design in Fig. 3, we note two observations. The first is the relative rate improvement of both schemes are almost the same for both design schemes. The second one is that the average $R_{\mathrm{s}}$ is less than its counterpart of the full CSI based design due to the lack of CSI.

Finally, we provide a comparison between the full-CSI and partial-CSI based designs using both PGS and IGS schemes. For this purpose, we compare the average $R_{\mathrm{S}}$ for both the IGS and the PGS based schemes versus $\overline{\mathcal{H}}_{\mathrm{p}}$ using full-CSI and partial CSI with $P_{\text {out,th }}=10^{-1}, 10^{-2}$ and $R_{\text {min }}=R_{\mathrm{t}}=4 \mathrm{bit} / \mathrm{sec} / \mathrm{Hz}$ in Fig. 6 . In addition, we compare between the throughput of the primary network using the PGS and the IGS based schemes for different CSI assumptions versus $\overline{\mathcal{H}}_{\mathrm{p}}$ in Fig. 7. First for the average $R_{\mathrm{s}}$ performance, it increases with the increase of $\overline{\mathcal{H}}_{\mathrm{p}}$ for both schemes PGS

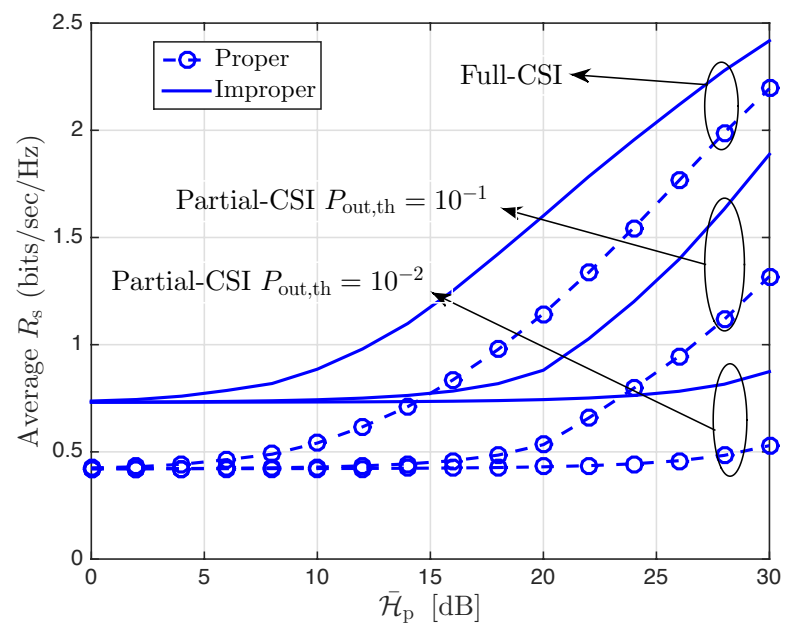

Fig. 6. Average $R_{\mathrm{S}}$ versus $\overline{\mathcal{H}}_{\mathrm{p}}$ for both PGS and IGS based schemes assuming full and partial CSI with $P_{\text {out,th }}=10^{-1}$ and $10^{-2}$.

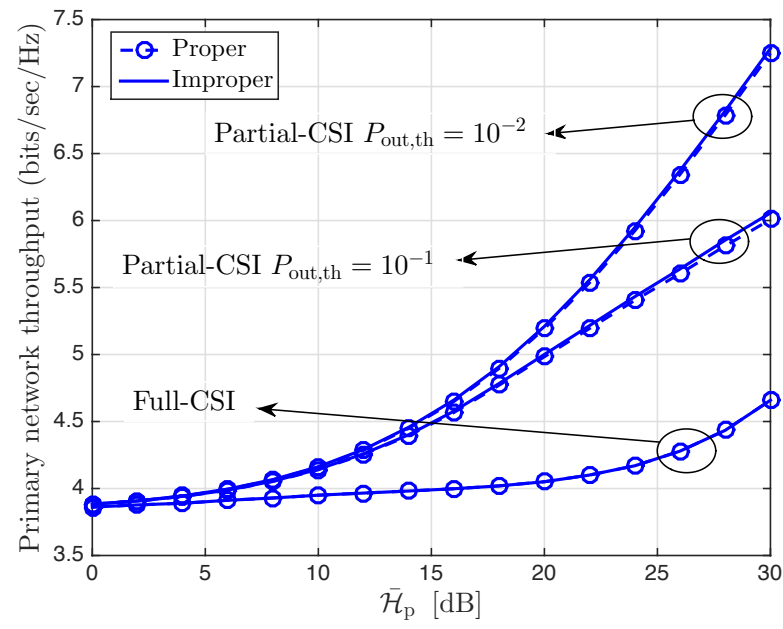

Fig. 7. The primary network throughput versus $\overline{\mathcal{H}}_{\mathrm{p}}$ for both PGS and IGS based schemes assuming full and partial CSI with $P_{\text {out,th }}=10^{-1}$ and $10^{-2}$.

and IGS. The availability of CSI gives an apparent gain of the average $R_{\mathrm{S}}$, while for partial CSI based design, the gain decreases by imposing more restriction on $P_{\text {out,th }}$. Moreover, the performance gap between the IGS and the PGS based schemes increases with the increase of $P_{\text {our,th }}$ in partialCSI based design. As for full-CSI based design, the gap decreases with the increase of $\overline{\mathcal{H}}_{\mathrm{p}}$, where the primary link becomes certainly good such that the relative interference impact decreases and the IGS solution tends to be near the PGS solution. As for the corresponding throughput performance of the primary network, better performance is achieved by the partial-CSI based design with lower $P_{\text {out,th. }}$. The lack of instantaneous CSI forces the overlay cognitive radio system to provide a design protection that reduces the room for secondary network rate improvement at a cost of improving the achievable rate of the primary network as can be shown in Fig. 7. 


\section{CONCLUSION}

In this paper, we studied the impact of employing the IGS scheme on designing the overlay cognitive radio with full CSI and partial CSI at the secondary network side. The ST divides its power between a proper signal for the primary network and an improper signal for the secondary network. We optimize the power allocation and the circularity coefficient to maximize the rate of the secondary network subject to a minimum rate requirement of the primary network. According to the derived optimal solution, the benefits of the IGS based scheme can be reaped when the channel gain of the interference link between the ST and the PR is more than the channel gain of the direct link of the secondary network. Moreover, the simulation results show that relative gain of adopting the IGS based scheme increases with the decrease of primary network direct link gain. The partial CSI based design provides extra protection on the primary network QoS at a cost of loosing rate improvement of the secondary network.

\section{APPENDIX A \\ DeCreasing Characteristics of $a\left(\mathcal{C}_{x}\right)$}

Define $z\left(\mathcal{C}_{x}\right)=1-\mathcal{C}_{x}^{2}\left(1-\frac{1}{2^{2 R_{\min }}}\right)$, then by evaluating $\frac{\partial a\left(\mathcal{C}_{x}\right)}{\partial \mathcal{C}_{x}}$, we obtain

$$
\frac{\partial a\left(\mathcal{C}_{x}\right)}{\partial \mathcal{C}_{x}}=\frac{1}{z^{2}\left(\mathcal{C}_{x}\right) e_{1} 2}\left(\frac{2+z\left(\mathcal{C}_{x}\right) e_{2}}{\sqrt{1+z\left(\mathcal{C}_{x}\right) e_{2}}}-2\right) \frac{\partial z\left(\mathcal{C}_{x}\right)}{\partial \mathcal{C}_{x}}
$$

where $e_{1}=p_{\mathrm{s}} \mathcal{G}_{\mathrm{s}}, e_{2}=\left(\frac{p_{\mathrm{s}} \mathcal{G}_{\mathrm{s}}+p_{\mathrm{p}} \mathcal{H}_{\mathrm{p}}+1}{2^{R_{\min }}}\right)^{2}-1$ and $\frac{\partial z\left(\mathcal{C}_{x}\right)}{\partial \mathcal{C}_{x}}$ is found to be

$$
\frac{\partial z\left(\mathcal{C}_{x}\right)}{\partial \mathcal{C}_{x}}=-2 \mathcal{C}_{x}\left(1-\frac{1}{2^{2 R_{\min }}}\right) .
$$

Since $z\left(\mathcal{C}_{x}\right)$ is positive for $0 \leq \mathcal{C}_{x} \leq 1$ and $R_{\min }>$ 0 , then $\frac{2+z\left(\mathcal{C}_{x}\right) e_{2}}{\sqrt{1+z\left(\mathcal{C}_{x}\right) e_{2}}}-2>0$ because $\left(2+z\left(\mathcal{C}_{x}\right) e_{2}\right)^{2}>$ $4\left(1+z\left(\mathcal{C}_{x}\right) e_{2}\right)$, where $e_{2}>0$ according to Theorem 1 . Therefore, $\frac{\partial a\left(\mathcal{C}_{x}\right)}{\partial \mathcal{C}_{x}}$ is a monotonically decreasing function since $\frac{\partial z\left(\mathcal{C}_{x}\right)}{\partial \mathcal{C}_{x}}<0$ as can be deduced from (65).

\section{APPENDIX B}

PROOF THAT $c(\alpha)$ IS REAL

First, to have a real $c(\alpha)$ value, we need to prove that

$$
\frac{\left(\left(1-\alpha_{\mathrm{IGS}}\right) p_{\mathrm{s}} \mathcal{G}_{\mathrm{s}}+1\right)^{2}}{\left(p_{\mathrm{s}} \mathcal{G}_{\mathrm{s}}+p_{\mathrm{p}} \mathcal{H}_{\mathrm{p}}+1\right)^{2}} 2^{2 R_{\min }}>1,
$$

which can be reduced after some manipulations to

$$
1+\frac{\alpha_{\text {IGS }} p_{\mathrm{s}} \mathcal{G}_{\mathrm{s}}+p_{\mathrm{p}} \mathcal{H}_{\mathrm{p}}}{\left(1-\alpha_{\mathrm{IGS}}\right) p_{\mathrm{s}} \mathcal{G}_{\mathrm{s}}+1}<2^{R_{\text {min }}}
$$

Since the PGS satisfy the primary link rate requirement, i.e.,

$$
1+\frac{\alpha_{\mathrm{PGS}} p_{\mathrm{s}} \mathcal{G}_{\mathrm{s}}+p_{\mathrm{p}} \mathcal{H}_{\mathrm{p}}}{\left(1-\alpha_{\mathrm{PGS}}\right) p_{\mathrm{s}} \mathcal{G}_{\mathrm{s}}+1}=2^{R_{\mathrm{min}}}
$$

and according to (19), we have $a\left(\mathcal{C}_{x}\right)<a(0)$, where $a(0)=$ $\alpha_{\mathrm{PGS}}$ for $\mathcal{C}_{x}>0$, then (66) is valid.

\section{APPENDIX C \\ EQUIVALENT RATE LIMITS FOR CASE 1}

In this section, we find the equivalent rate limits of $0<$ $\tilde{\alpha} \leq \alpha_{\mathrm{PGS}}$ in terms of $R_{\mathrm{min}}$. Since $\tilde{\alpha} \leq \alpha_{\mathrm{PGS}}$ is equivalent to $R_{\min } \geq R_{\mathrm{I}, 1}$, while $\tilde{\alpha}>0$, can be simplified obtaining

$$
2^{2 R_{\min }}<\left(1+p_{\mathrm{s}} \mathcal{H}_{\mathrm{s}}\right)\left(\frac{p_{\mathrm{s}} \mathcal{G}_{\mathrm{s}}}{p_{\mathrm{s}} \mathcal{G}_{\mathrm{s}}-p_{\mathrm{s}} \mathcal{H}_{\mathrm{s}}}\right),
$$

which reduces to $R_{\min }<R_{\mathrm{I}, 3}$. Therefore, we find that $0<$ $\tilde{\alpha} \leq \alpha_{\mathrm{PGS}}$ is equivalent to

$$
R_{\mathrm{I}, 1} \leq R_{\min }<R_{\mathrm{I}, 3} .
$$

As for $c(\tilde{\alpha}) \geq 1$, it can be simplified obtaining

$$
\begin{aligned}
\left(2(1-\tilde{\alpha}) p_{\mathrm{s}} \mathcal{G}_{\mathrm{s}}+1\right) 2^{2 R_{\min }}+ & \left((1-\tilde{\alpha}) p_{\mathrm{s}} \mathcal{G}_{\mathrm{s}}\right)^{2} \geq \\
& \left(p_{\mathrm{s}} \mathcal{G}_{\mathrm{s}}+p_{\mathrm{p}} \mathcal{H}_{\mathrm{p}}+1\right)^{2},
\end{aligned}
$$

then by using (22) and substitute in (71), we obtain

$$
\begin{aligned}
2 \times 2^{4 R_{\min }}\left(\frac{p_{\mathrm{s}} \mathcal{G}_{\mathrm{s}}}{p_{\mathrm{s}} \mathcal{H}_{\mathrm{s}}}-1\right) & -\frac{p_{\mathrm{s}} \mathcal{G}_{\mathrm{s}}}{p_{\mathrm{s}} \mathcal{H}_{\mathrm{s}}} 2^{2 R_{\min }}-\frac{p_{\mathrm{s}} \mathcal{G}_{\mathrm{s}}}{p_{\mathrm{s}} \mathcal{H}_{\mathrm{s}}} \\
& -\left(p_{\mathrm{s}} \mathcal{G}_{\mathrm{s}}+p_{\mathrm{p}} \mathcal{H}_{\mathrm{p}}+1\right)^{2} \geq 0 .
\end{aligned}
$$

After finding the roots of the left-hand-side, then we obtain $R_{\text {min }} \leq R_{\mathrm{I}, 2}$.

As a result, from (70), the Case 1 conditions in (30) reduces to the following expression that is equivalent to (31)

$$
\left(\alpha_{\mathrm{IGS}}, \mathcal{C}_{x}^{*}\right)= \begin{cases}(\tilde{\alpha}, c(\tilde{\alpha})) & R_{\min }<R_{\mathrm{I}, 2}, R_{\mathrm{I}, 1}<R_{\min } \leq R_{\mathrm{I}, 3} \\ (a(1), 1) & R_{\min } \geq R_{\mathrm{I}, 2}, R_{\mathrm{I}, 1}<R_{\min } \leq R_{\mathrm{I}, 3}\end{cases}
$$

\section{APPENDIX D \\ EQUIVALENT RATE LIMITS FOR CASE 2}

From $a(1) \leq 0$, we obtain

$$
\left(\frac{1}{2^{2 R_{\min }}} p_{\mathrm{s}} \mathcal{G}_{\mathrm{s}}+1\right)^{2} \leq 1+\frac{1}{2^{2 R_{\min }}}\left(\left(\frac{p_{\mathrm{s}} \mathcal{G}_{\mathrm{s}}+p_{\mathrm{p}} \mathcal{H}_{\mathrm{p}}+1}{2^{R_{\min }}}\right)^{2}-1\right),
$$

which can be simplified to $R_{\min } \leq R_{\mathrm{I}, 4}$. Since $\tilde{\alpha} \leq 0$, then we get $R_{\min } \geq R_{\mathrm{I}, 3}$. As a result, we obtain the following expression that is equivalent to (33).

$$
\left(\alpha_{\mathrm{IGS}}, \mathcal{C}_{x}^{*}\right)=\left\{\begin{array}{ll}
(a(1), 1) & R_{\min } \geq R_{\mathrm{I}, 4} \text { and } R_{\min } \geq R_{\mathrm{I}, 3} \\
(0, c(0)) & R_{\mathrm{min}} \leq R_{\mathrm{I}, 4} \text { and } R_{\min } \geq R_{\mathrm{I}, 3}
\end{array},\right.
$$

\section{REFERENCES}

[1] Q. Zhao and B. M. Sadler, "A survey of dynamic spectrum access," IEEE Signal Process. Mag., vol. 24, no. 3, pp. 79-89, May 2007.

[2] S. Srinivasa and S. A. Jafar, "Cognitive radios for dynamic spectrum access-the throughput potential of cognitive radio: A theoretical perspective," IEEE Commun. Mag., vol. 45, no. 5, pp. 73-79, May 2007.

[3] F. D. Neeser and J. L. Massey, "Proper complex random processes with applications to information theory," IEEE Trans. Inf. Theory, vol. 39, no. 4, pp. 1293-1302, Jul. 1993.

[4] P. J. Schreier and L. L. Scharf, Statistical signal processing of complexvalued data: the theory of improper and noncircular signals. Cambridge University Press, 2010.

[5] V. R. Cadambe, S. A. Jafar, and C. Wang, "Interference alignment with asymmetric complex signaling-settling the Høst-MadsenNosratinia conjecture," IEEE Trans. Inf. Theory, vol. 56, no. 9, pp. 4552-4565, Sep. 2010. 
[6] Y. Zeng, C. M. Yetis, E. Gunawan, Y. L. Guan, and R. Zhang, "Transmit optimization with improper Gaussian signaling for interference channels," IEEE Trans. Signal Process., vol. 61, no. 11, pp. 2899-2913, Jun. 2013.

[7] Y. Zeng, R. Zhang, E. Gunawan, and Y. Guan, "Optimized transmission with improper Gaussian signaling in the K-user MISO interference channel," IEEE Trans. Wireless Commun., vol. 12, no. 12, pp. 63036313, Dec. 2013.

[8] M. Gaafar, M. G. Khafagy, O. Amin, and M.-S. Alouini, "Improper gaussian signaling in full-duplex relay channels with residual selfinterference," in Proc. IEEE Int. Conf. Commun. (ICC), Kuala Lumpure, May. 2016.

[9] M. Gaafar, O. Amin, A. Ikhlef, A. Chaaban, and M.-S. Alouini, "On alternate relaying with improper gaussian signaling," IEEE Commun. Lett., vol. 20, no. 8, pp. 1683-1686, Aug. 2016.

[10] C. Lameiro, I. Santamaría, and P. J. Schreier, "Benefits of improper signaling for underlay cognitive radio," IEEE Wireless Commun. Lett., vol. 4, no. 1, pp. 22-25, Feb. 2015.

[11] C. Lameiro, I. Santamaria, and P. Schreier, "Analysis of maximally improper signaling schemes for underlay cognitive radio networks," in Proc. IEEE Int. Conf. Commun. (ICC). London: IEEE, 2015, pp. 13981403.

[12] O. Amin, W. Abediseid, and M.-S. Alouini, "Outage performance of cognitive radio systems with improper Gaussian signaling," in Proc. IEEE Int. Symp. on Information Theory (ISIT), Hong Kong, Jun. 2015.

[13] _ - "Underlay cognitive radio systems with improper gaussian signaling: Outage performance analysis," IEEE Trans. Wireless Commun., vol. 15, no. 7, Jul. 2016.

[14] M. Gaafar, O. Amin, W. Abediseid, and M.-S. Alouini, "Spectrum sharing opportunities of full-duplex systems using improper Gaussian signaling," in in Proc. IEEE Int. Symp. on Personal, Indoor and Mobile Radio Communications (PIMRC), Hong Kong, Sep. 2015.

[15] — "Sharing the licensed spectrum of full-duplex systems using improper Gaussian signaling," in in Proc. IEEE Global Communincation Conf. (GLOBECOM), San Diego, Dec. 2015.

[16] M. Gaafar, O. Amin, W. Abediseid, and M. S. Alouini, "Underlay spectrum sharing techniques with in-band full-duplex systems using improper Gaussian signaling," to appear in IEEE Trans. Wireless Commun., DOI: 10.1109/TWC.2016.2621767 2016.

[17] A. Jovičić and P. Viswanath, "Cognitive radio: An information-theoretic perspective," IEEE Trans. Inf. Theory, vol. 55, no. 9, pp. 3945-3958, Sep. 2009.

[18] C. Hellings, L. Gerdes, L. Weiland, and W. Utschick, "On optimal Gaussian signaling in MIMO relay channels with partial decode-andforward," IEEE Trans. Signal Process., vol. 62, no. 12, pp. 3153-3164, Jun. 2014

[19] W. Su, J. D. Matyjas, and S. Batalama, "Active cooperation between primary users and cognitive radio users in heterogeneous ad-hoc networks," IEEE Trans. Signal Process., vol. 60, no. 4, pp. 1796-1805, Apr. 2012.

[20] P. Bhagawat, W. Wang, M. Uppal, G. Choi, Z. Xiong, M. B. Yeary, and A. Harris, "An FPGA implementation of dirty paper precoder." in Proc. IEEE Int. Conf. Commun. (ICC), Glasgow, 2007, pp. 2761-2766.

[21] M. H. M. Costa, "Writing on dirty paper," IEEE Trans. Inf. Theory, vol. 29, no. 3, pp. 439-441, May 1983.

[22] J. Lee, Y. Kim, H. Lee, B. L. Ng, D. Mazzarese, J. Liu, W. Xiao, and Y. Zhou, "Coordinated multipoint transmission and reception in LTEadvanced systems," IEEE Commun. Mag., vol. 50, no. 11, pp. 44-50, Nov. 2012.

[23] S. Ghorpade and B. V. Limaye, A course in multivariable calculus and analysis. Springer, 2010.

[24] V. V. Prasolov, Polynomials. Springer Science \& Business Media, 2009.

[25] S. Neumark, Solution of cubic and quartic equations. Elsevier, 2014.

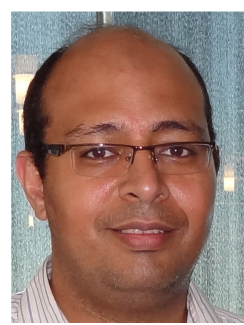

Osama Amin (S'07, M'11, SM'15) received B. Sc. degree in Electrical and Electronics Engineering from Aswan University, Aswan, Egypt, in 2000, M. Sc. degree in Electrical Engineering from Assiut University, Assiut, Egypt in 2004 and Ph.D. degree in Electrical and Computer Engineering, University of Waterloo, Canada in 2010. In June 2012, he joined Assiut University as an Assistant Professor in the Electrical and Electronics Engineering department. Currently, he is with King Abdullah University of Science and Technology (KAUST), Thuwal, Makkah, Kingdom of Saudi Arabia. His general research interests lie in communications systems and signal processing for communications with special emphasis on wireless applications. Specific research areas include green communications, cognitive radio, cooperative communications, and channel estimation.

Dr. Amin is an Editor for the IEEE Communications Letters. He has served as a technical program committee (TPC) member for ICC, IEEE VTC, CROWNCOM, PIMRC, and ISSPIT conferences. He has served also as co-organizer and a co-chair of the Next Generation Green ICT and 5G Networking (GreeNets) 2015 in the IEEE International Conference on Ubiquitous Wireless Broadband (ICUWB) in Montreal, Canada.

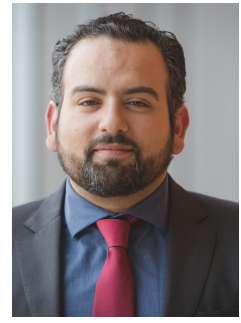

Walid Abediseid (S'04, M'11) was born in Etobicoke, Ontario, Canada. He received the B. Sc. and M. Sc. degrees in Electrical Engineering from the University of Ottawa, Canada, in 2004 and 2007, respectively. He then received his Ph.D. from the Department of Electrical and Computer Engineering, University of Waterloo in 2011. He was a postdoctoral fellow at King Abdullah University of Science and Technology (KAUST), Thuwal, Makkah Province, Saudi Arabia, from December 2011 to December 2013. His research interests included coding and information theory, MIMO wireless communication systems, lattice applications for digital and wireless communications, detection and estimation.

In January 2014, Dr. Abediseid joined the Saudi Initiatives department at KAUST where he holds the academic development specialist of the Saudi Research Science Institute. He also serves as a tutor during the SRSI summer program that provides support to students conducting research in STEM fields.

Dr. Abediseid was a recipient of many prestigious awards during his graduate studies such as the Research In Motion Graduate Scholarship from 2008 to 2009, and the NSERC Alexander Graham Bell Canada Graduate Scholarshipone of Canada's most prestigious graduate awards from 2009 to 2011.

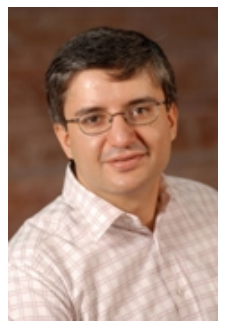

Mohamed-Slim Alouini (S'94, M'98, SM'03, F'09) was born in Tunis, Tunisia. He received the Ph.D. degree in Electrical Engineering from the California Institute of Technology (Caltech), Pasadena, CA, USA, in 1998. He served as a faculty member in the University of Minnesota, Minneapolis, MN, USA, then in the Texas A\&M University at Qatar, Education City, Doha, Qatar before joining King Abdullah University of Science and Technology (KAUST), Thuwal, Makkah Province, Saudi Arabia as a Professor of Electrical Engineering in 2009. His current research interests include the modeling, design, and performance analysis of wireless communication systems. 